\title{
Lead Discovery for Alzheimer's Disease Related Target Protein RbAp48 from Traditional Chinese Medicine
}

\author{
Hung-Jin Huang, ${ }^{1}$ Cheng-Chun Lee, ${ }^{2}$ and Calvin Yu-Chian Chen ${ }^{2,3}$ \\ ${ }^{1}$ Department of Chinese Pharmaceutical Sciences and Chinese Medicine Resources, College of Pharmacy, China Medical University, \\ Taichung 40402, Taiwan \\ ${ }^{2}$ School of Medicine, College of Medicine, China Medical University, Taichung 40402, Taiwan \\ ${ }^{3}$ Department of Biomedical Informatics, Asia University, Taichung 41354, Taiwan
}

Correspondence should be addressed to Cheng-Chun Lee; leeck@mail.cmu.edu.tw and Calvin Yu-Chian Chen; ycc929@mit.edu

Received 14 February 2014; Accepted 1 March 2014; Published 2 June 2014

Academic Editor: Chung Y. Hsu

Copyright (C) 2014 Hung-Jin Huang et al. This is an open access article distributed under the Creative Commons Attribution License, which permits unrestricted use, distribution, and reproduction in any medium, provided the original work is properly cited.

\begin{abstract}
Deficiency or loss of function of Retinoblastoma-associated proteins (RbAp48) is related with Alzheimer's disease (AD), and AD disease is associated with age-related memory loss. During normal function, RbAp48 forms a complex with the peptide FOG-1 (friend of GATA-1) and has a role in gene transcription, but an unstable complex may affect the function of RbAp48. This study utilizes the world's largest traditional Chinese medicine (TCM) database and virtual screening to provide potential compounds for RbAp48 binding. A molecular dynamics (MD) simulation was employed to understand the variations after protein-ligand interaction. FOG1 was found to exhibit low stability after RbAp48 binding; the peptide displayed significant movement from the initial docking position, a phenomenon which matched the docking results. The protein structure of the other TCM candidates was not variable during MD simulation and had a greater stable affinity for RbAp48 binding than FOG1. Our results reveal that the protein structure does not affect ligand binding, and the top three TCM candidates Bittersweet alkaloid II, Eicosandioic acid, and Perivine might resolve the instability of the RbAp48-FOG1 complex and thus be used in AD therapy.
\end{abstract}

\section{Introduction}

Alzheimer's disease $(\mathrm{AD})$ is the most common neurodegenerative disease to occur in people around the ages of 65 to 69 years [1], but it is not a normal part of aging and younger people may also suffer from $\mathrm{AD}$ disease [2-4], although these cases are not common. AD symptoms involve memory loss, cognitive impairment which affects the ability to study, a reduction in activities, feeling loss, and long-term memory loss. The major neuropathology hallmarks are deposition of neuritic plaques and neurofibrillary tangles in the AD brain $[5,6]$. Genetic mutations are the known causes of AD disease [7], with mutations occurring inthe genes for the amyloid precursor protein (APP). Presenilin 1 and presenilin 2 (PS1 and PS2) enhance the processing of transmembrane APP cleaved by alpha and beta proteases and gamma-secretases to form beta-amyloid 42 [8], which subsequently results in the development of $\mathrm{AD}$. In curing this disease, in animal models, antiamyloid therapies were used to clear amyloid accumulation. However, recent strategies have not been successful in human AD patients. Scientists still do not fully understand the causes of AD disease, because of the existence of more than one high risk factor for neuronal dysfunction. In recent studies, Pavlopoulos et al. have demonstrated that Retinoblastoma-associated protein (RbAp48) deficiency or loss of function in the dentate gyrus (DG) is related to agerelated memory loss [9]. RbAp48, which is a member of the $\mathrm{NuRD}$ (nucleosome remodeling and deacetylase) complex, is a histone-binding protein that targets chromatin assembly factors. NuRD is associated with gene expression and the presence of histone deacetylases for regulating transcription repressors $[10,11]$. The transcription activation and repression of NuRD is regulated by FOG-1 (friend of GATA-1) which binds to RbAp48.

Traditional Chinese medicine (TCM) has been developed in China over thousands of years, and includes herbal 


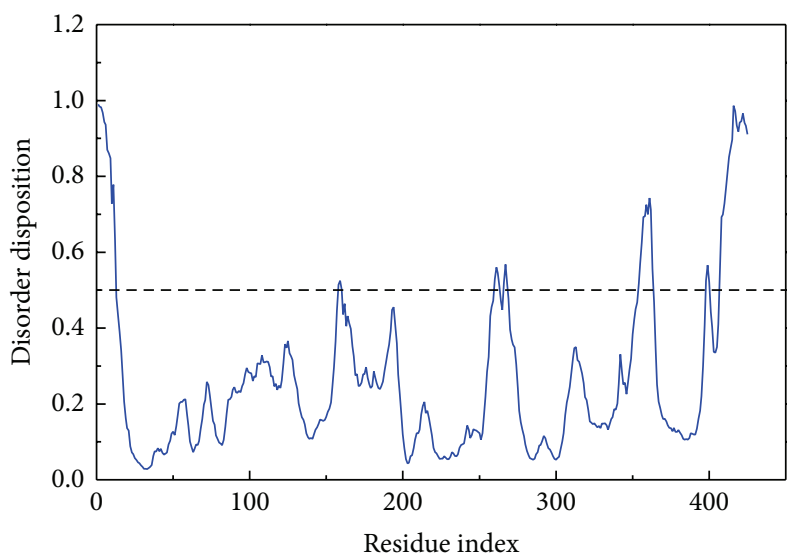

FIGURE 1: Disorder analysis of sequence of RbAp48 from the result of PONDR-FIT prediction. A value of disorder disposition above 0.5 in disorder disposition indicates disorder residues.

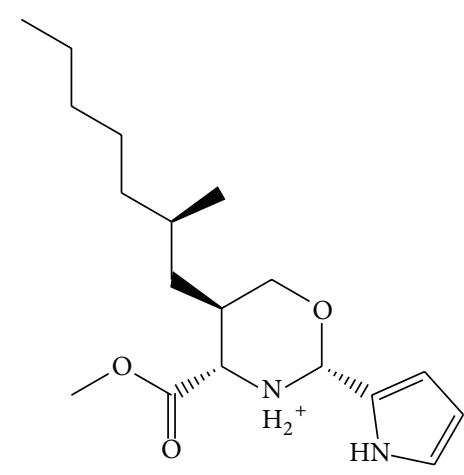

Bittersweet alkaloid II

(a)

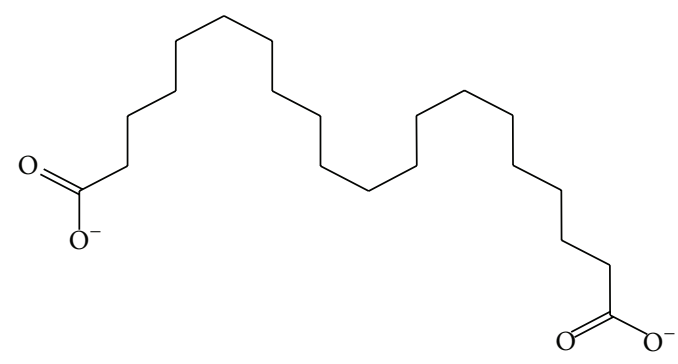

Eicosandioic acid

(b)

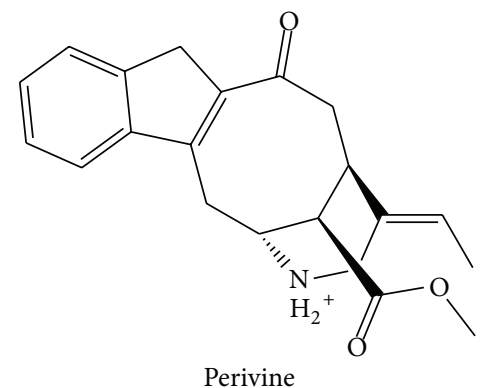

(c)

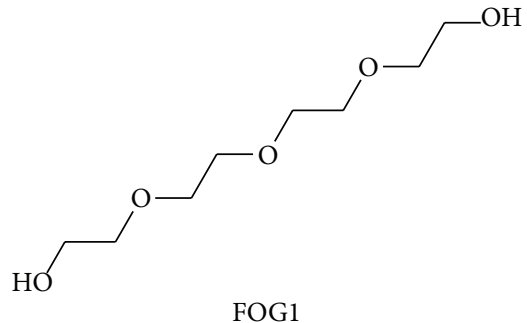

(d)

Figure 2: Chemical scaffolds of TCM candidates and control.

medicine, acupuncture, Cupping, and Qigong. Traditional Chinese medicine has been used for stroke prevention [1214], and in the treatment of cancer [15]. In this research, computer-aided drug design (CADD) is utilized. CADD has been widely used in many drug design studies $[16,17]$ which include molecular modeling approaches [18] and web server calculation $[19,20]$. TCM is widely used in clinical treatment because of low side effects and low toxicity [21-23], and some studies have used extracts of Chinese herbs to investigate the therapeutic value of potential drugs [24, 25]. Research of CADD and TCM has been performed in many studies, such as influenza therapy [26-28], stroke prevention [29, 30], treatment of erectile dysfunction [31], reducing weight [32, 33], type II diabetes therapy [34], diseases associated with aging treatment [35], inflammation inhibitors development [36], HIV treatment [22], Parkinson's disease prevention [37], and cancer therapy $[12,38,39]$. Hence, we present a small molecule from the world's largest TCM database [40] to bind to $\mathrm{RbAp} 48$ and provide a more potent compound for target protein (RbAp48) binding than FOG-1. 


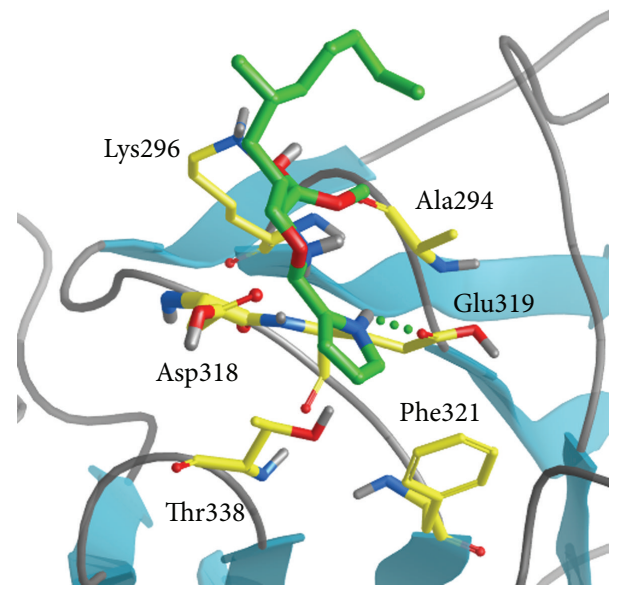

(a)

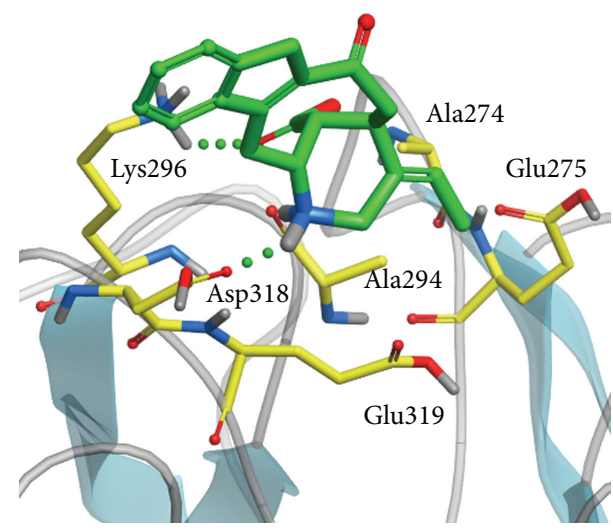

(c)

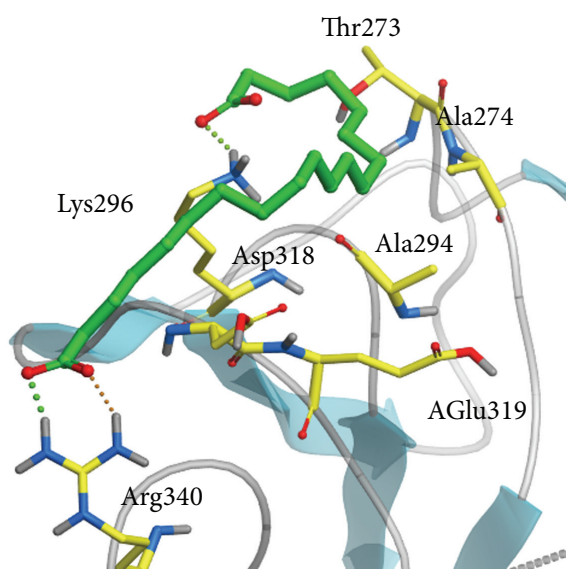

(b)

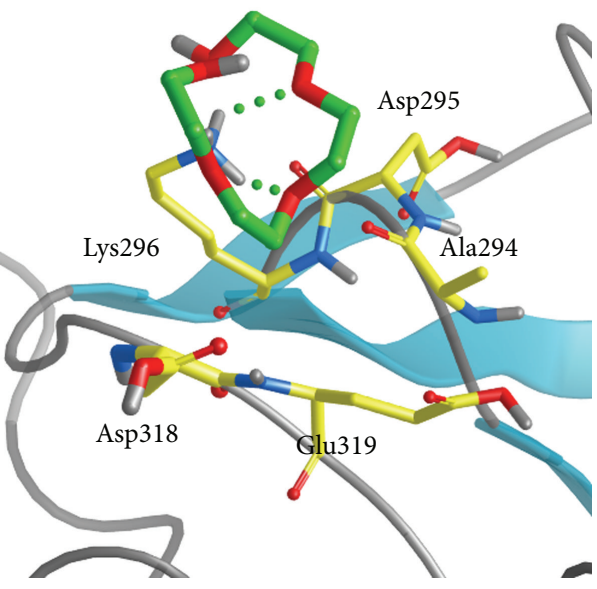

(d)

FIGURE 3: The docking poses of small compounds: (a) Bittersweet alkaloid II, (b) Eicosandioic acid, (c) Perivine, and (d) FOG1 in binding site of RbAp48. The small molecular and amino acids are colored in green and yellow, respectively.

\section{Materials and Methods}

2.1. Protein Preparation. The crystal structure of RbAp48 was taken from the PDB database (PDB code: 3RIK) [41]; the missing atoms and protonation were cleaned up by Prepare Protein module under Accelrys Discovery Studio 2.5.5.9350 (DS 2.5) [42]. All residues were protonated at $\mathrm{pH}$ 7.4. For structure validation, PONDR-FIT [43] was carried out to predict unfolded regions on the RbAp48 sequence.

2.2. Docking Analysis. Database screening involved 61,000 TCM compounds from the TCM Database@Taiwan (http://tcm.cmu.edu.tw/) being used for docking analysis, with the drug-likeness of all compounds being assessed by ADMET prediction. The LigandFit module in DS 2.5 was utilized to generate different ligand poses by Monte-Carlo techniques. All the ligand conformations were minimized by the CHARMm force field. The Ligand-receptor interaction energies were calculated by the DREIDING force field during the docking progress. Minimization was performed on 1,000 steps of Steepest Descent and then minimized by Conjugate Gradient. Different generated ligands poses were docked into the defined binding site on the RbAp48 protein structure; ligand binding in the receptor cavity was evaluated by various scoring functions, including -PMF, -PMF04, and Dock Score.

2.3. Molecular Dynamics Simulation. The protein-ligand complexes were regarded as input structures in GROMACS 4.5.5 package [44] for molecular dynamic simulation; charmm 27 force field was selected in the simulation system. The distance of the real space for box definition was set as $1.2 \mathrm{~nm}$. The particle mesh Ewald (PME) method was used to treat Coulomb interactions as electrostatic. The Coulomb interaction between two charge particles was as follows:

$$
V_{c}\left(r_{i j}\right)=f \frac{q_{i} q_{j}}{\varepsilon_{r} r_{i j}} .
$$




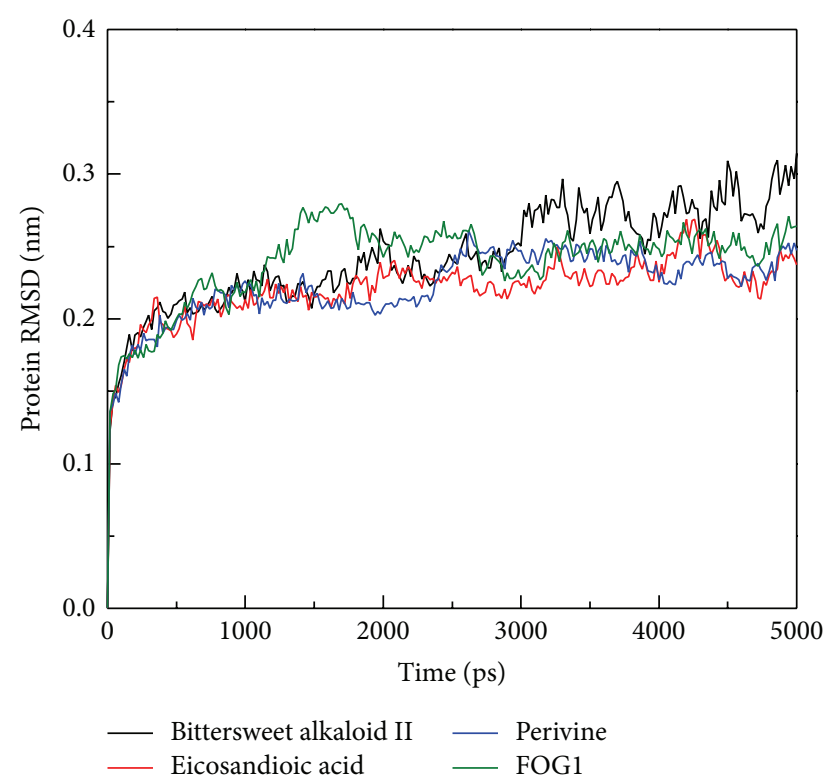

(a)

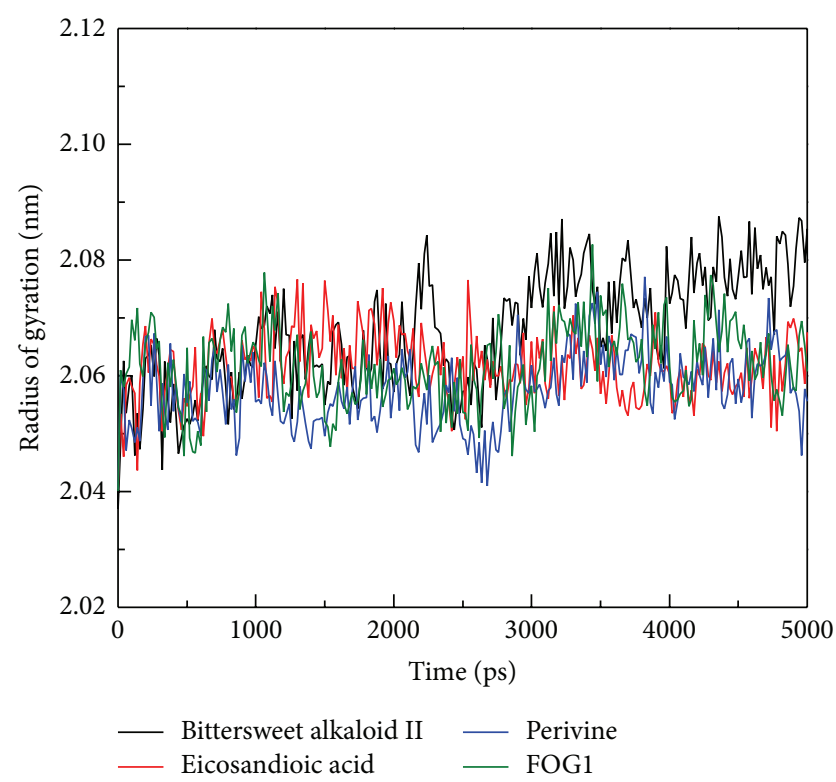

(b)

FIGURE 4: Plots of (a) Protein RMSD and (b) radius of gyration from protein-ligand complexes for a simulation time of 5,000 ps.

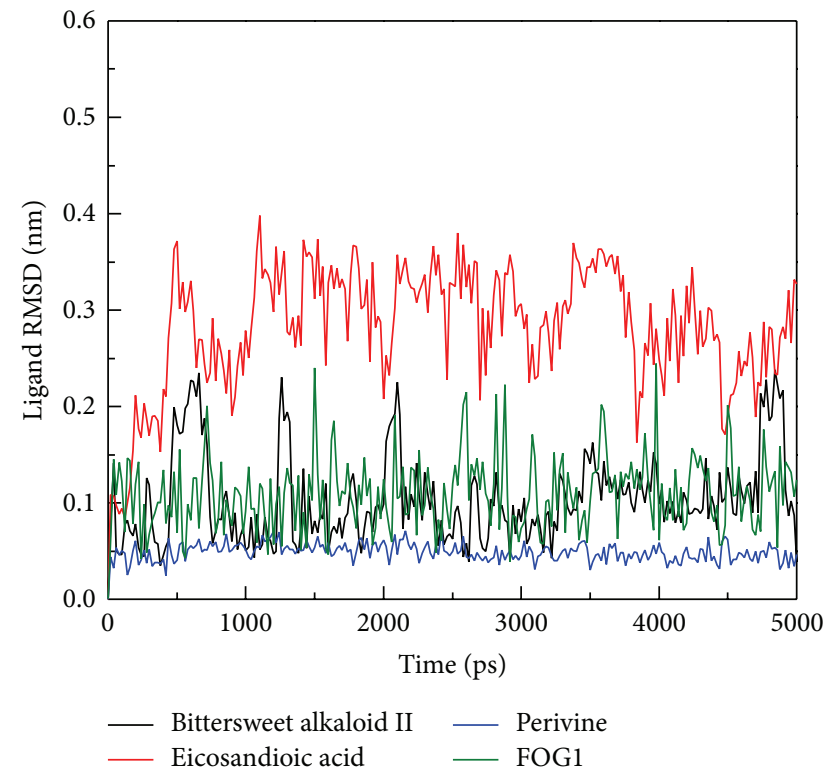

Figure 5: Plot of Ligand RMSD values from protein-ligand complexes for a simulation time of 5,000 ps.

The cut-off distance of van der Waals (VDW) residues was set at $1.4 \mathrm{~nm}$, using the following equation:

$$
U(r)=4 \varepsilon\left[\left(\frac{\delta}{\gamma}\right)^{12}-\left(\frac{\delta}{\gamma}\right)^{6}\right] .
$$

The linear constraint solver (LINCS) algorithm was used for fixing all bond lengths. The solvent setting for water simulation was based on the TIP3P model. Topology files and parameters of small compounds for docked ligands were generated by SwissParam web server [45]. We added $\mathrm{Na}$ and $\mathrm{Cl}$ ions to create a neutral system; the concentration of $\mathrm{NaCl}$ model was set to $0.145 \mathrm{M}$. 5,000 cycle steps of the steepest descent algorithm were used for energy minimization then followed by equilibration performed under position restraints for $1 \mathrm{~ns}$ under constant temperature dynamics (NVT type) conditions at a temperature of $310 \mathrm{~K}$. Following this step, all production dynamics simulations were performed for 5,000 ps under constant pressure and temperature dynamics (NPT type). The temperature of the simulation system was set as $310 \mathrm{~K}$. MD conformations were saved every 20 ps for trajectory, migration, and residues fluctuation analysis.

2.4. Molecular Dynamics Analysis. All MD conformations were analyzed under GROMACS 4.5.5 software, root mean square deviation (RMSD), and radius of gyration (Rg) by the commands g_cluster and g_gyrate, respectively. Total energy was calculated by the g_energy program. Root mean squared fluctuation (RMSF) of protein residues was obtained by g_rmsf. Mean square displacement (MSD) was performed using g_msd; the docked ligand was used to observe the migration over the simulation time. Cluster analysis was performed to the cluster docked ligand complex by g_cluster program. The method for cluster determination was the linkage algorithm.

\section{Results and Discussion}

3.1. Docking Results. We employed PONDR-FIT [43] to predict the order/disorder from the protein sequence, in order to understand if the binding region of RbAp48 is an order-folded structure. The sequence number of the major 


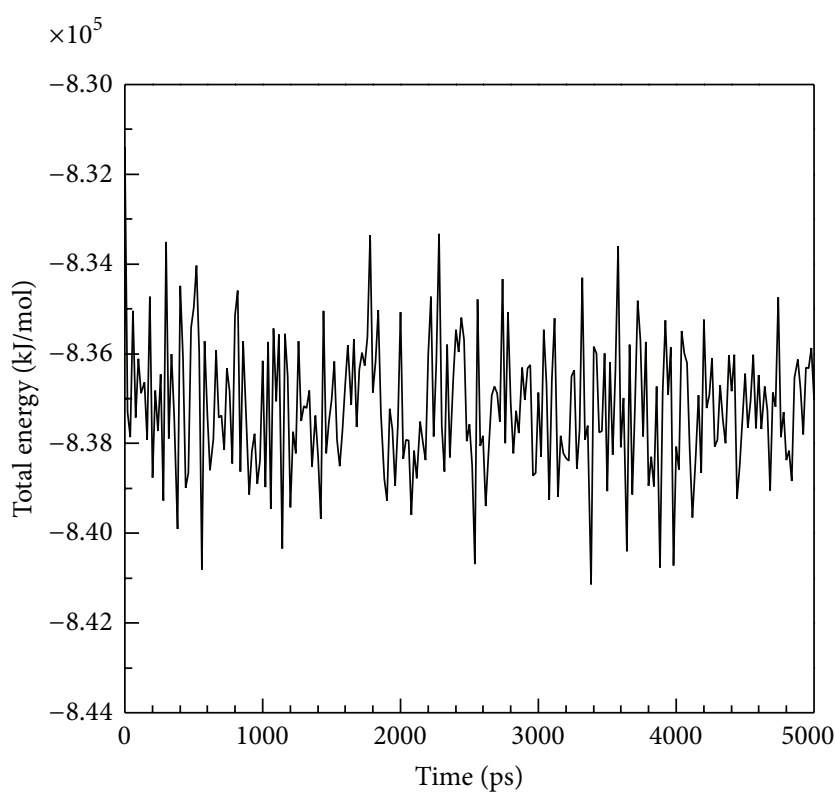

— Bittersweet alkaloid II

(a)

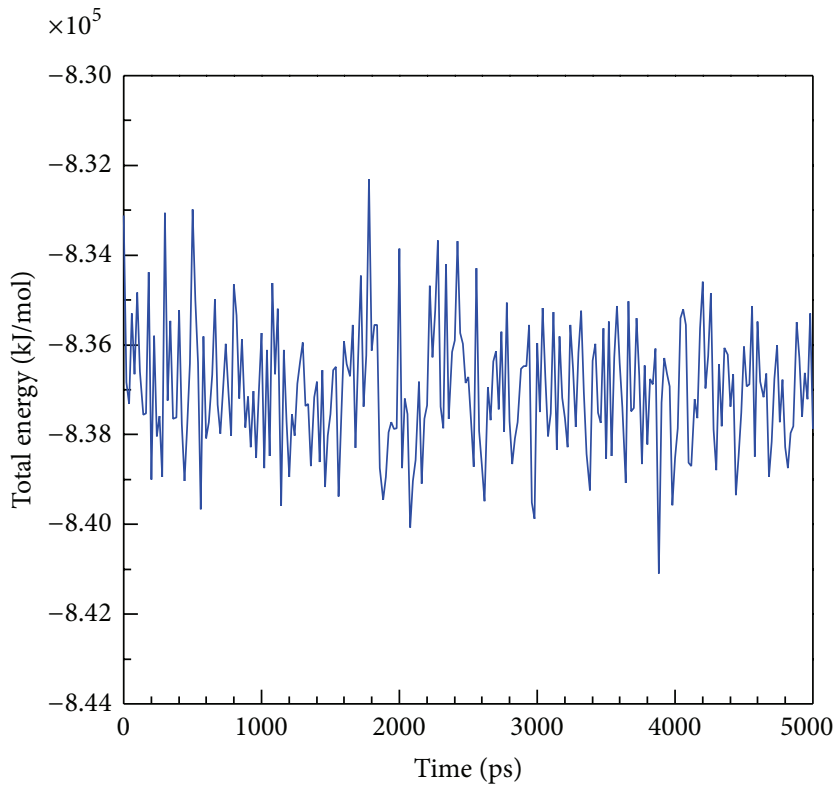

— Perivine

(c)

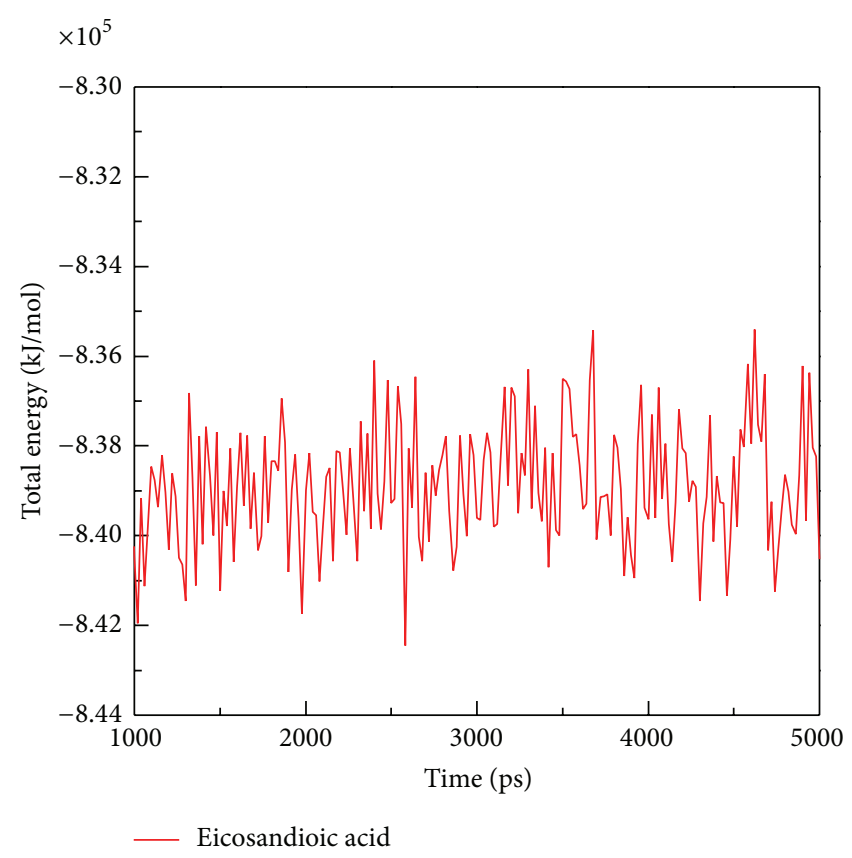

(b)

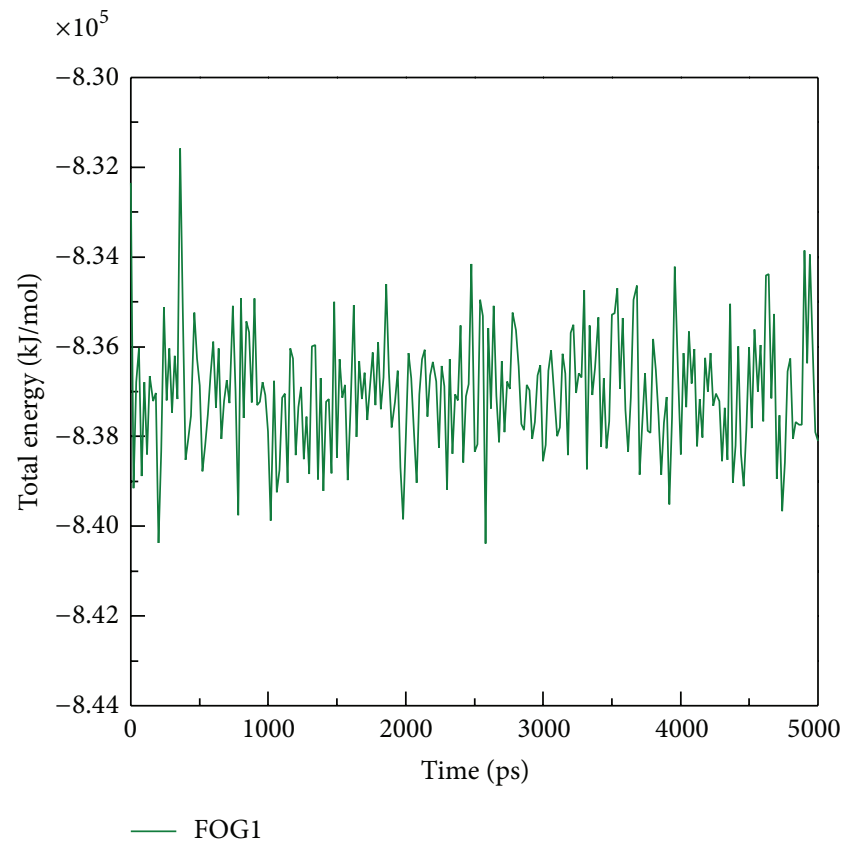

(d)

Figure 6: Total energy of RbAp48 with docked ligand: (a) Bittersweet alkaloid II, (b) Eicosandioic acid, (c) Perivine, and (d) FOG1 for a simulation time of 5,000 ps.

binding region was from 250 to 350 (Figure 1), and the values for disorder disposition were below 0.5 , which indicated that the binding site of RbAp48 is a folded structure, and the ligand binding may not be affected by protein structure [46]. Docking analysis was based on -PMF, -PMF04, and Dock Score to evaluate the docking pose of traditional Chinese medicine (TCM) compounds. From the scoring analysis, FOG1 was regarded as a control for comparison; candidates with higher values of scores than FOG1 are shown in Table 1. For ADMET evaluation, all the TCM candidates and FOG1 had no CYP2D6 inhibition; suggesting that CYP2D6 may not be affected by these ligands in the liver. The top TCM candidates displayed good absorption (absorption = 0 ), high or medium blood brain barrier (BBB) penetration (penetration $=1$ or 2$)$, and good drug-like solubility $(-4.0$ $<$ solubility value $<-2.0)$. FOG1 had moderate absorption 


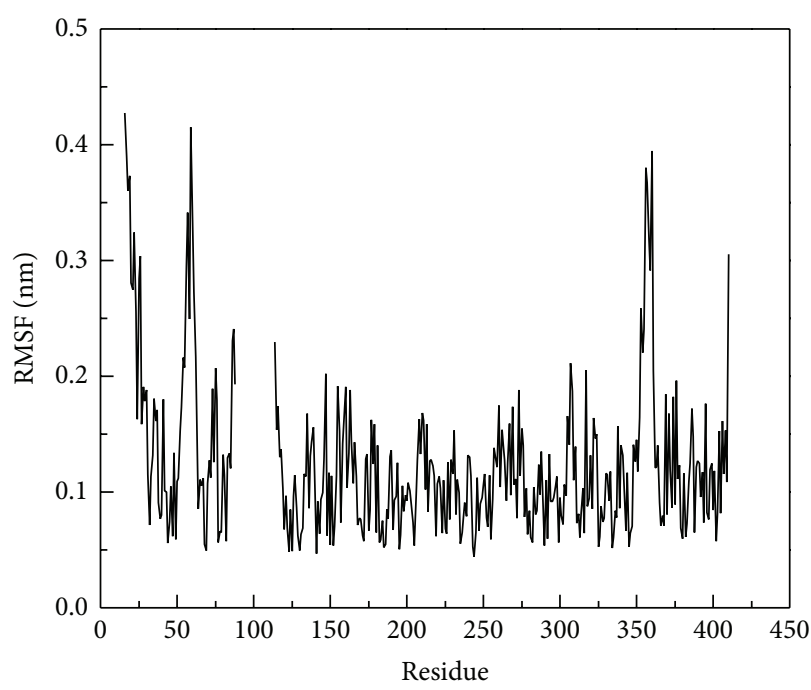

— Bittersweet alkaloid II

(a)

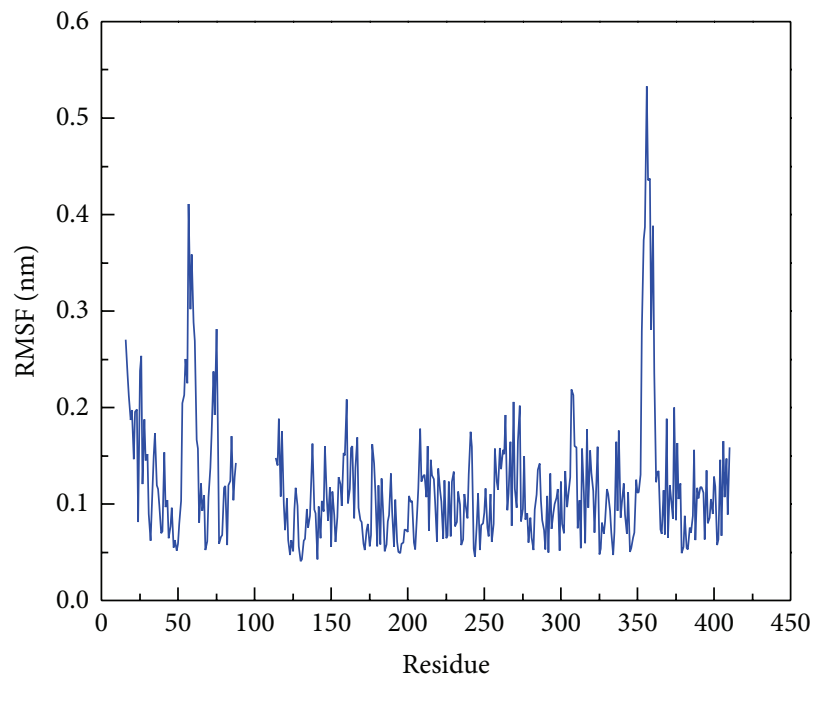

— Perivine

(c)

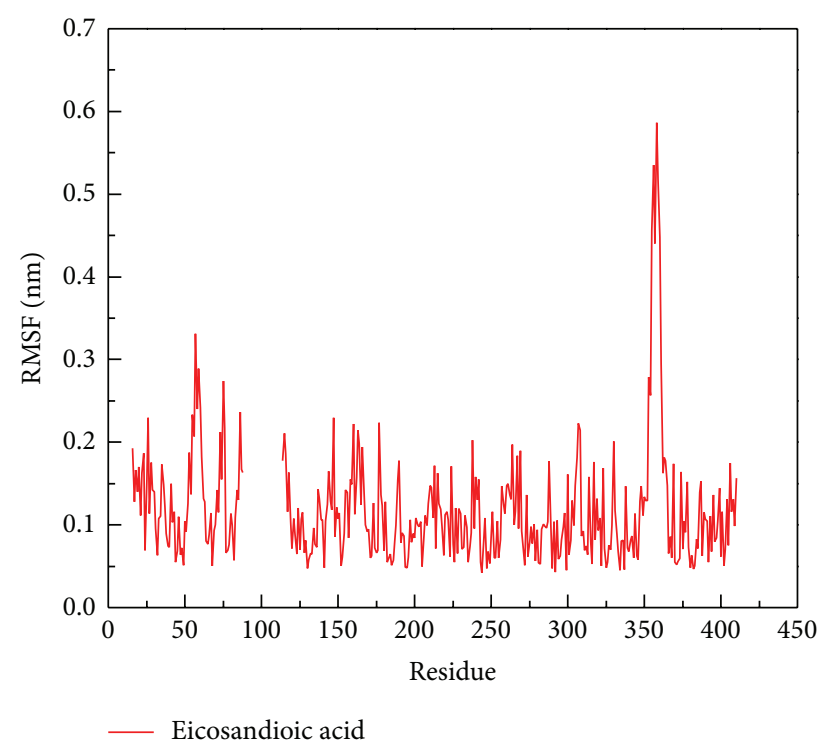

(b)

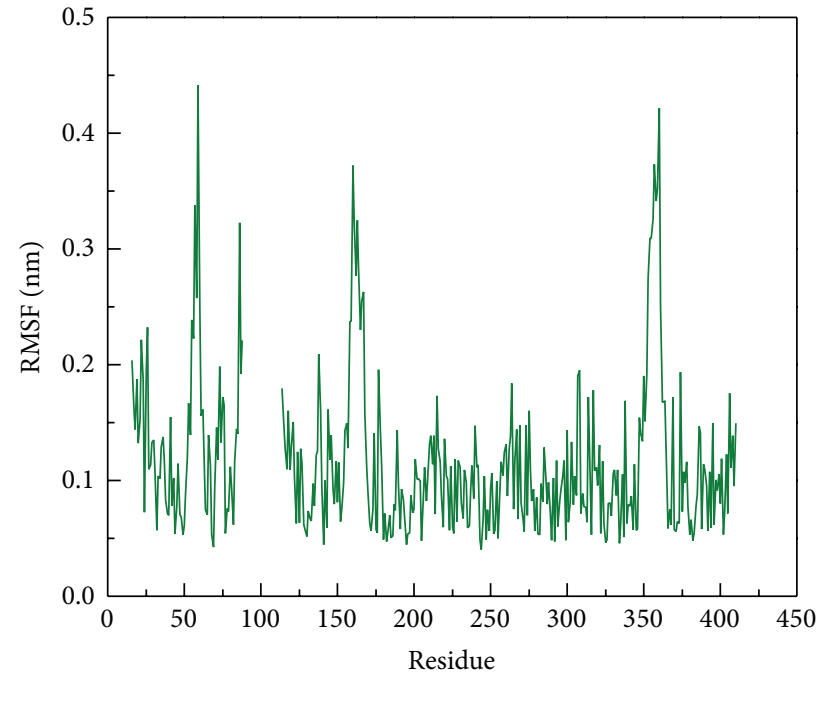

(d)

FIGURE 7: RMSF values of each residue of RbAp48 with docked ligand: (a) Bittersweet alkaloid II, (b) Eicosandioic acid, (c) Perivine, and (d) FOG1 ligand for a simulation time of 5,000 ps.

(absorption $=1)$, undefined BBB penetration (penetration $=$ $4)$, and low drug-likeness absorption $(-6.0<$ solubility value $<-4.0$ ). These data show that the top TCM candidates are more drug-like than the control. All docked ligands were ranked by Dock Score, and it was found that the Dock Score of the top TCM candidates (including score values of -PMF and -PMF04) was greater than FOG1. Furthermore, due to the score value of -PMF04 varying significantly between Perivine and Docosandioic acid, we selected Bittersweet alkaloid II, Eicosandioic acid, and Perivine for further study. The chemical scaffolds of the TCM candidates and the control are shown in Figure 2. Docking poses of Bittersweet alkaloid II displayed $\mathrm{H}$-bond with Glu319; close residues include
Lys296, Asp318, Thr388, Phe321, and Ala294 (Figure 3(a)). For Eicosandioic acid, there are two amino acids (Arg340 and Asp295) which form H-bond interactions with the ligand; the surrounding residues are Asp318, Glu319, Ala294, Ala274, and Thr273 (Figure 3(b)). Perivine has two amino acids that generate H-bonds for ligand binding: Lys296 and Asp318; the amino acids Glu319, Glu275, Ala294, and Ala274 are near the docked ligand (Figure 3(c)). For the docked pose of FOG1, only Lys 296 of RbAp48 can generate H-bond interactions; close residues include Asp318, Glu319, Ala294, and Asp295 (Figure 3(d)). It is worth noting that Ala294 is the common residue for each ligand binding, and the result reveals that all the small compounds were bound in the same region of 


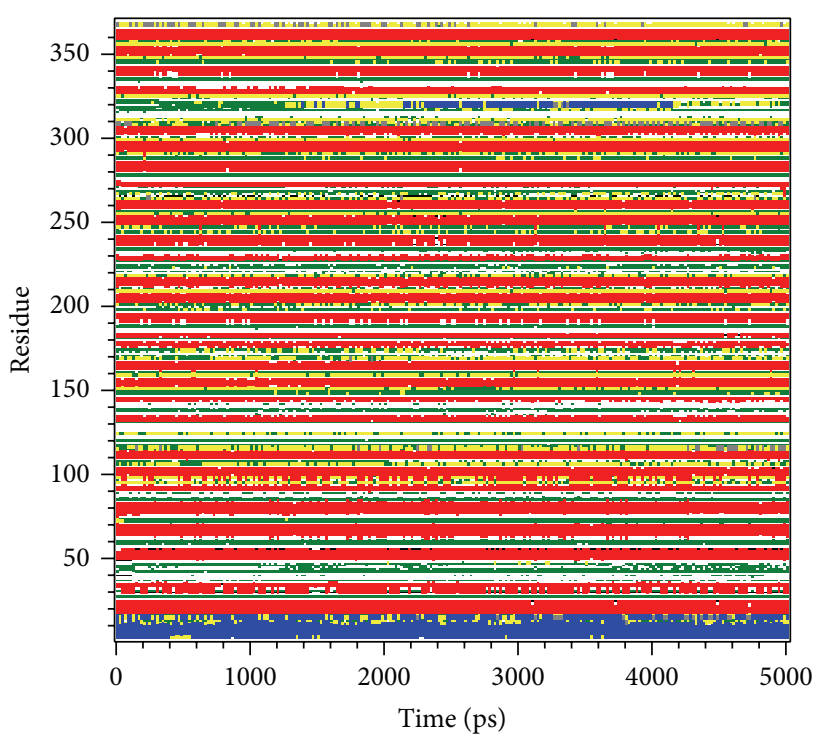

(a)

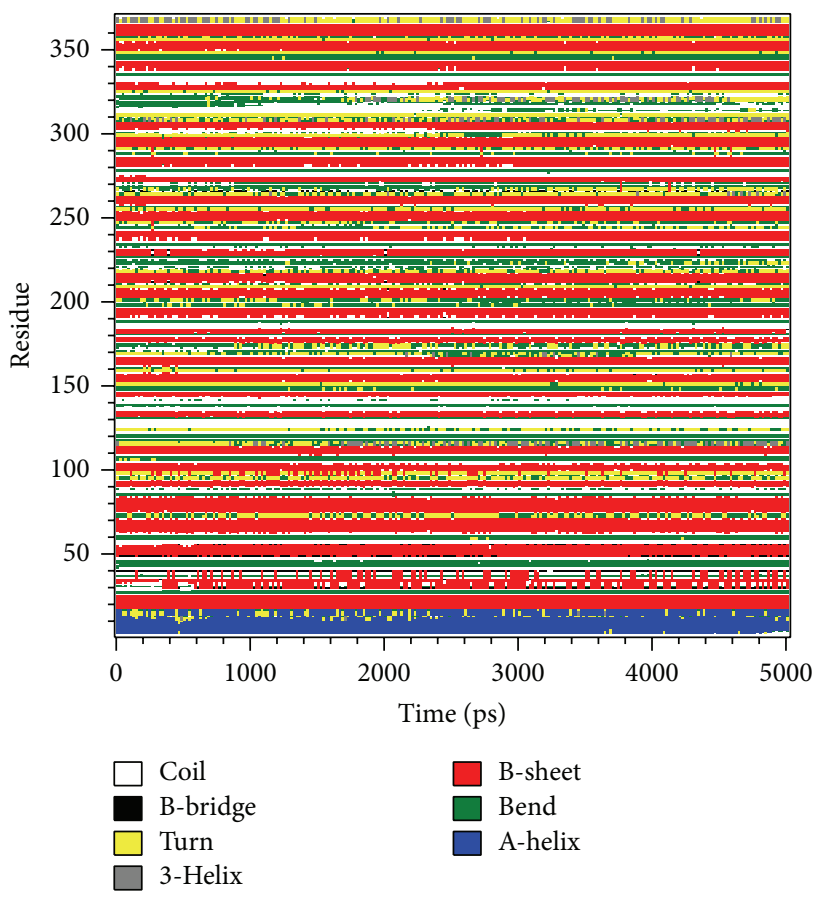

(c)

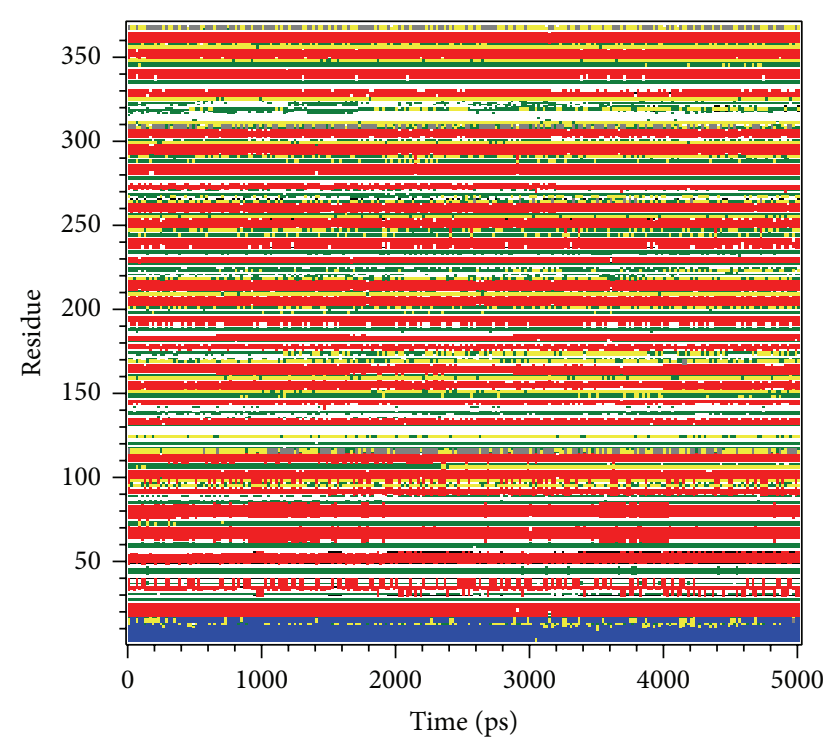

(b)

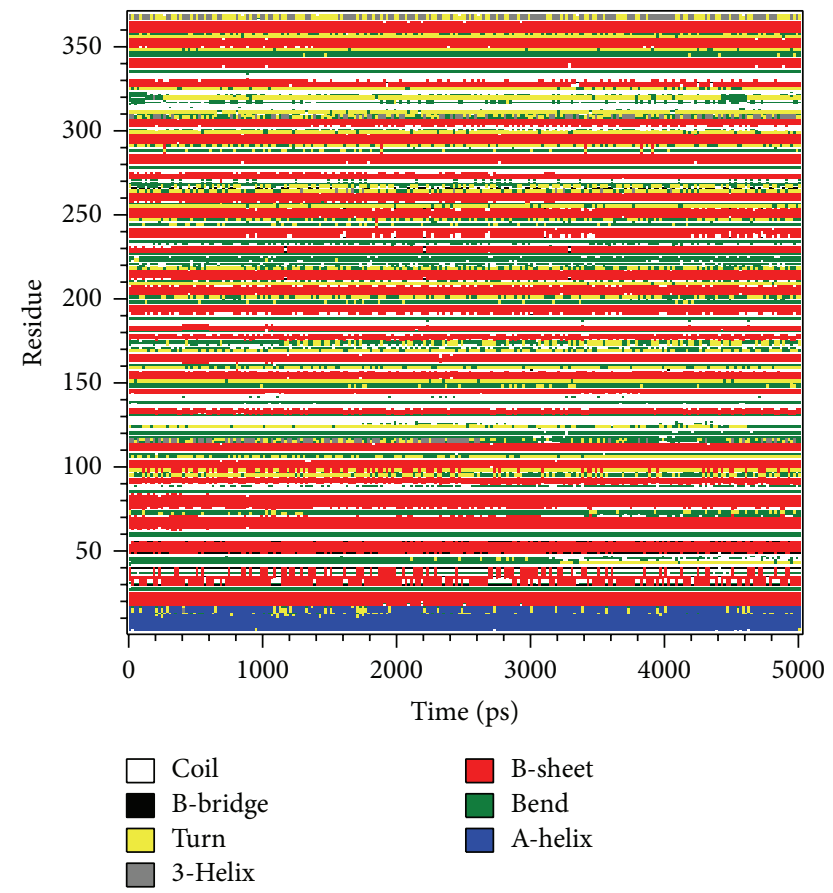

(d)

FIGURE 8: DSSP analysis of complexes with ligands: (a) Bittersweet alkaloid II, (b) Eicosandioic acid, (c) Perivine, and (d) FOG1.

RbAp48. In a further study, molecular dynamics simulation was utilized to analyze the variation of each ligand in the protein structures.

3.2. Stability Analysis of the Dynamics Complexes. The RMSD value of protein atoms and the $\mathrm{Rg}$ value were used to analyze the stability of the protein structure. The value of protein RMSD was between 0.2 and $0.3 \mathrm{~nm}$ from 1,000 to $5,000 \mathrm{ps}$ (Figure 4(a)); substantial fluctuations were not observed indicating that all conformations are stable after a simulation time of 1,000 ps. For Rg's plot evaluation, the complex with
Bittersweet alkaloid II is slightly increased from 2,000 to $5,000 \mathrm{ps}$, but the $\mathrm{Rg}$ value does not move away from the initial value (Figure 4(b)). The value for the Rg complex with Eicosandioic acid, Perivine, and FOG1 remained constant during a simulation time of $5,000 \mathrm{ps}$, which revealed that the protein structure is compact after MD simulation. Bittersweet alkaloid II may affect the structure of RbAp48, but Rg's plot shows that the complex remained stable from 3,000 to 5,000 ps.

The RMSD of each small molecule during MD simulation (Figure 5) was also analyzed, and the Ligand RMSD of 


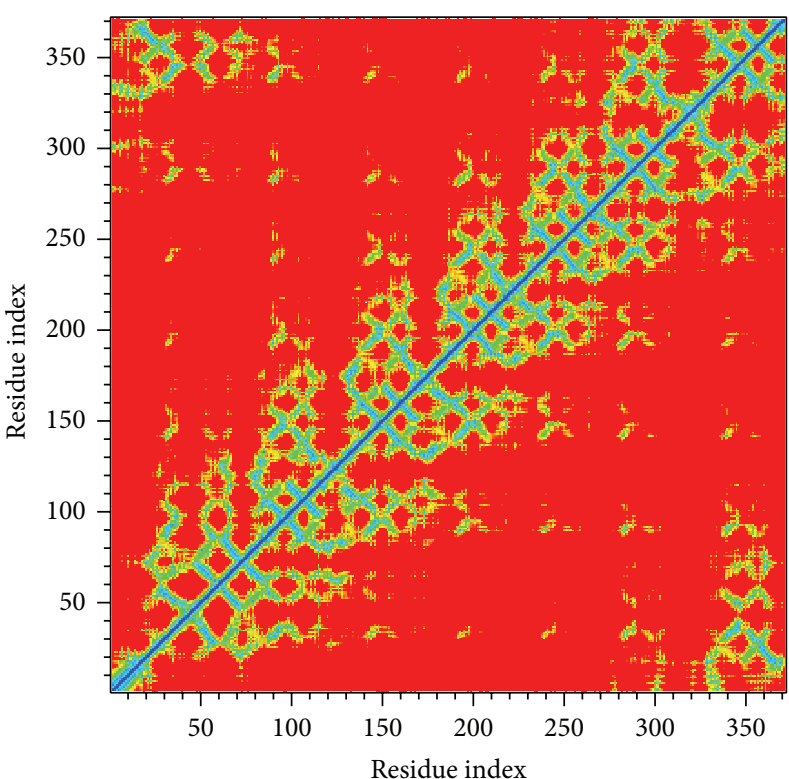

(a)

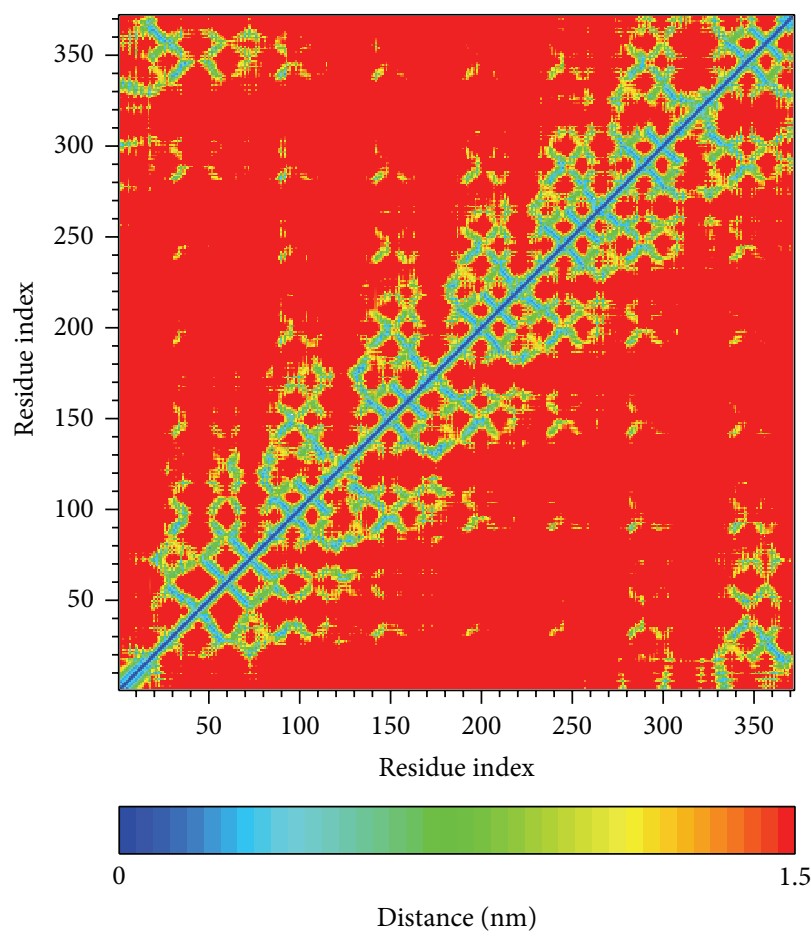

(c)

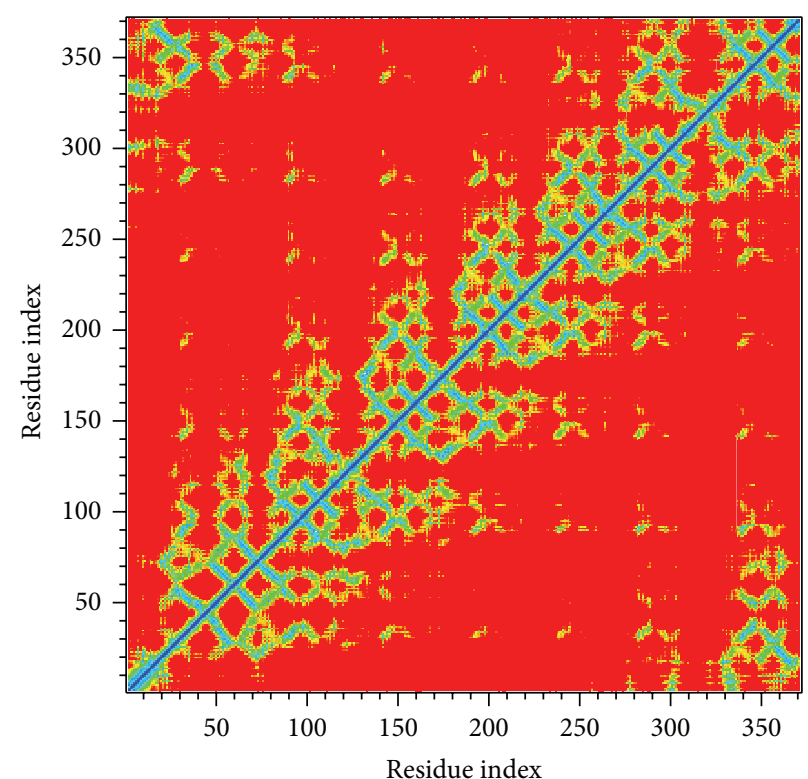

(b)

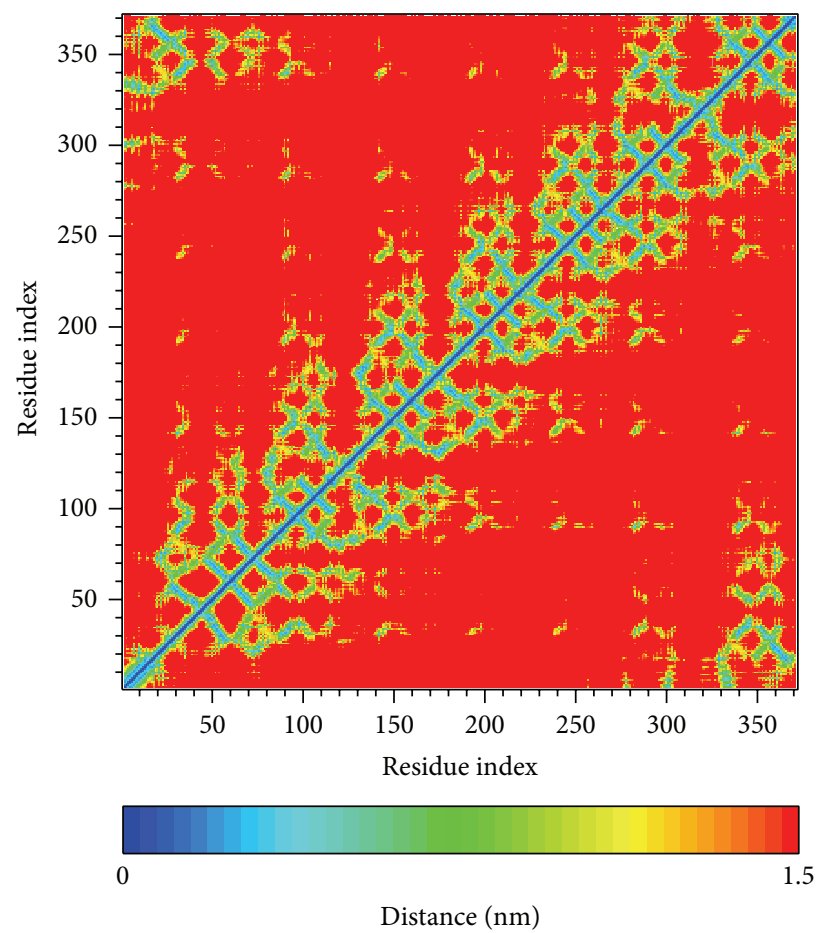

(d)

FIGURE 9: Matrix of smallest distance between each pair of amino acids in complex with (a) Bittersweet alkaloid II, (b) Eicosandioic acid, (c) Perivine, and (d) FOG1.

Eicosandioic acid was found to show large fluctuations at $500 \mathrm{ps}$ and tended to an average of $0.25 \mathrm{~nm}$ from $500 \mathrm{ps}$ to the end of the simulation. Bittersweet alkaloid II had similar fluctuations to FOG1, with an average of 0.1. The most stable ligand RMSD was found in Perivine, which was constant among all MD simulations. No significantly increased values were observed for total energy analysis in any complexes during a simulation time of $5,000 \mathrm{ps}$ (Figure 6). The total energy remained around $-8.35 \times 10^{5} \mathrm{~kJ} / \mathrm{mol}$ for Bittersweet alkaloid II, Perivine, and FOG1. In contrast, Eicosandioic acid had the lowest total energy, the average being -8.39 $\times 10^{5} \mathrm{~kJ} / \mathrm{mol}$. These results suggest that all structures of the complexes remained constant during MD simulation, and the binding regions do not display structural flexibility. 
TABLE 1: Top ten candidates and control.

\begin{tabular}{|c|c|c|c|c|c|c|c|}
\hline Name & -PMF & -PMF04 & Dock score & Absorption $^{\mathrm{a}}$ & BBB level $^{\mathrm{b}}$ & CYP2D6 $^{\mathrm{c}}$ & Solubility $^{\mathrm{d}}$ \\
\hline Bittersweet alkaloid II & 48.19 & 36.70 & 98.838 & $\mathbf{0}$ & 2 & $\mathbf{0}$ & 3 \\
\hline Eicosandioic acid & 48.52 & 20.37 & 97.934 & $\mathbf{0}$ & 2 & $\mathbf{0}$ & 3 \\
\hline Perivine & 30.39 & 23.90 & 95.328 & $\mathbf{0}$ & 2 & $\mathbf{0}$ & 3 \\
\hline Docosandioic acid & 34.37 & 17.84 & 95.194 & 0 & 1 & 0 & 3 \\
\hline N-Methylmescaline & 33.25 & 30.41 & 93.733 & 0 & 2 & 0 & 4 \\
\hline Isolobelanine & 38.74 & 22.15 & 93.190 & 0 & 1 & 0 & 3 \\
\hline 5-Methoxy-N-methyltryptamine & 24.72 & 22.30 & 89.676 & 0 & 2 & 0 & 4 \\
\hline Vincamine & 31.63 & 28.02 & 89.338 & 0 & 2 & 0 & 3 \\
\hline Quininone & 35.22 & 26.12 & 88.540 & 0 & 2 & 0 & 3 \\
\hline FOG1 $^{*}$ & 4.65 & 14.08 & 67.647 & 1 & 4 & $\mathbf{0}$ & 5 \\
\hline
\end{tabular}

${ }^{*}$ Control.

a Absorption: good absorption: 0; moderate absorption: 1; low absorption: 2.

${ }^{b}$ BBB level (blood brain barrier): high penetration: 1; medium penetration: 2; low penetration: 3; undefined penetration: 4.

${ }^{\mathrm{c} C Y P 2 D 6:}$ noninhibitor $<0.5$; inhibitor $>0.5$.

${ }^{\mathrm{d}}$ Solubility: $-6.0<$ value $<-4.0$ indicates low drug-likeness compounds; $-4.0<$ value $<-2.0$ indicates good drug-likeness compounds; $-2.0<$ value $=0.0$ indicates optimal drug-likeness compounds; $0.0<$ value indicates being too soluble and with no drug-likeness.

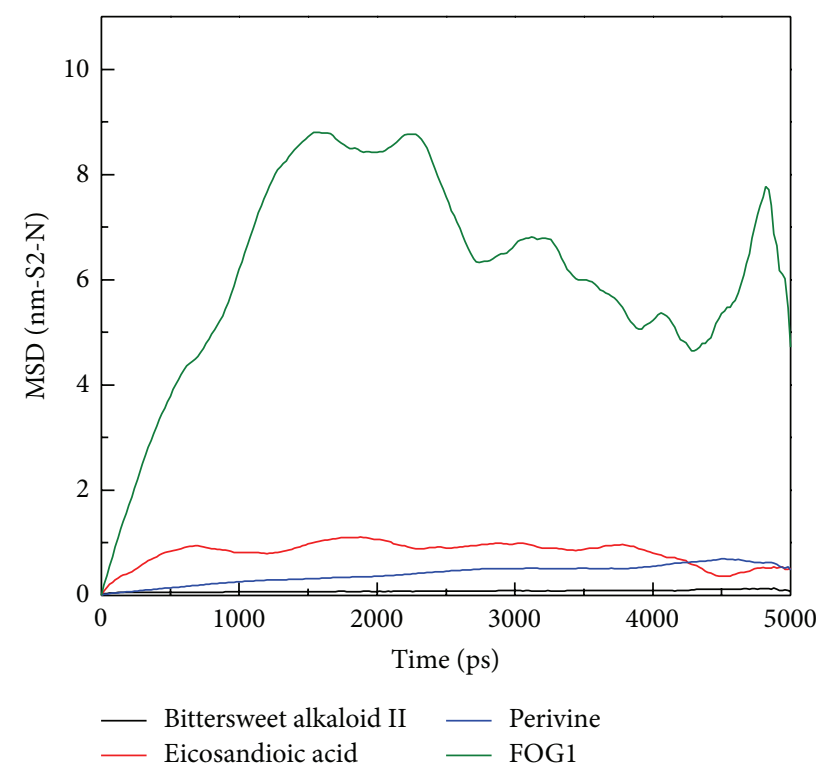

FIGURE 10: Mean square displacement (MSD) of different ligands for a simulation time of 5,000 ps. The value of MSD indicates migration of ligand from the initial site.

3.3. Stability Analysis of Residues on the Major Binding Region during MD Simulation. We calculated the RMSF of each residue to analyze the flexibility of residues on protein structure. The major binding region (from 250 to 350 residues) showed no significant increment in structure of RbAp48 with all ligands (Figure 7). From DSSP analysis, all complexes remained exist helices and beta-sheets during a simulation time of 5,000 ps (Figure 8). We also calculated the distance per pair of each residue for 5,000 ps. The matrix of smallest distance between each pair of amino acids showed that there were no distinct changes for all protein-ligand complexes (Figure 9). The results reveal that the structure of RbAp48 remained stable during all MD simulations.

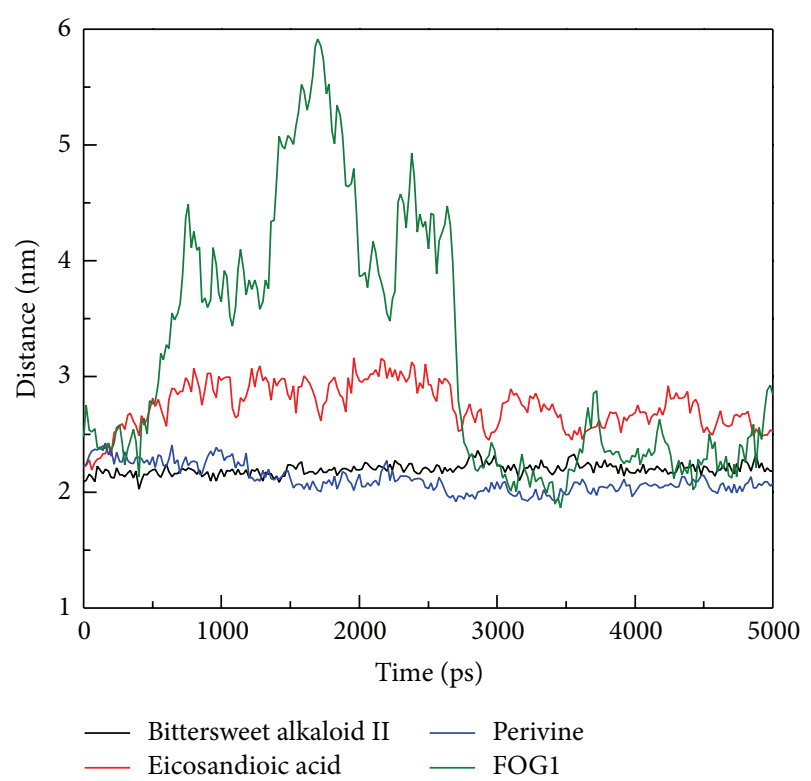

FIGURE 11: Distance between centers of mass of RbAp48 and each ligand for a simulation time of 5,000 ps.

3.4. Migration Analysis of Ligands in Protein Binding Site. MSD was used to measure the migration of the docked ligand during MD simulation in order to assess the variation of each ligand after docking into the protein binding site. The MSD value of FOG1 was the most distinctive and displayed a rapid increase during initial simulation to the end of the 5,000 ps (Figure 10). All TCM candidates still had an MDS value below $1 \mathrm{~nm}$. These results suggest that the docking poses were not changing the binding position significantly during the simulation time. In further study, the distances between the center mass of the protein and each ligand were measured for all simulation times to understand the movement of the docked compounds. Interestingly, FOG1 was found to 

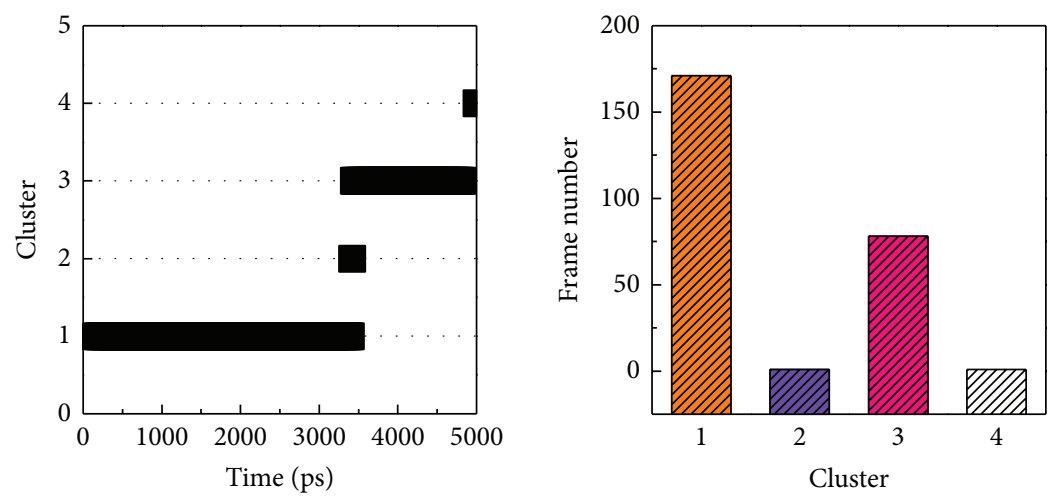

(a)
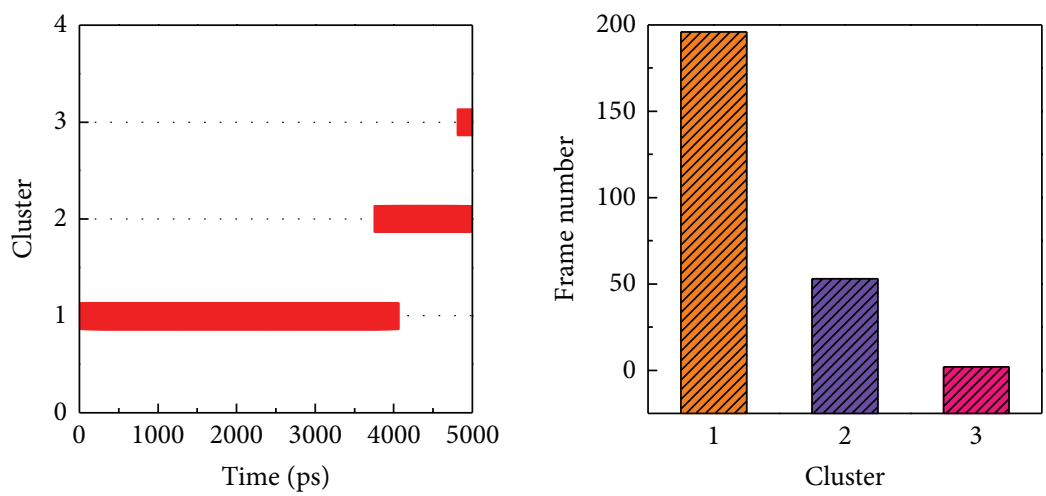

(b)
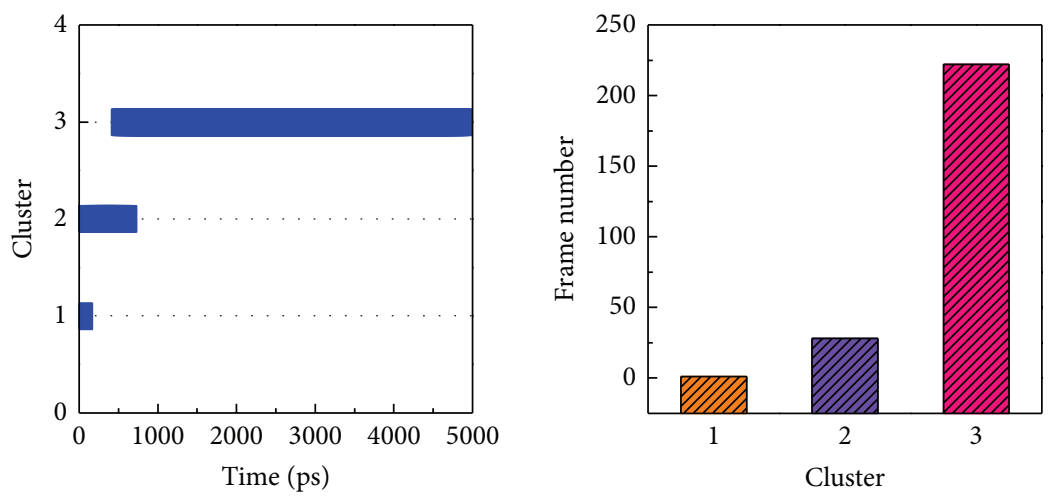

(c)
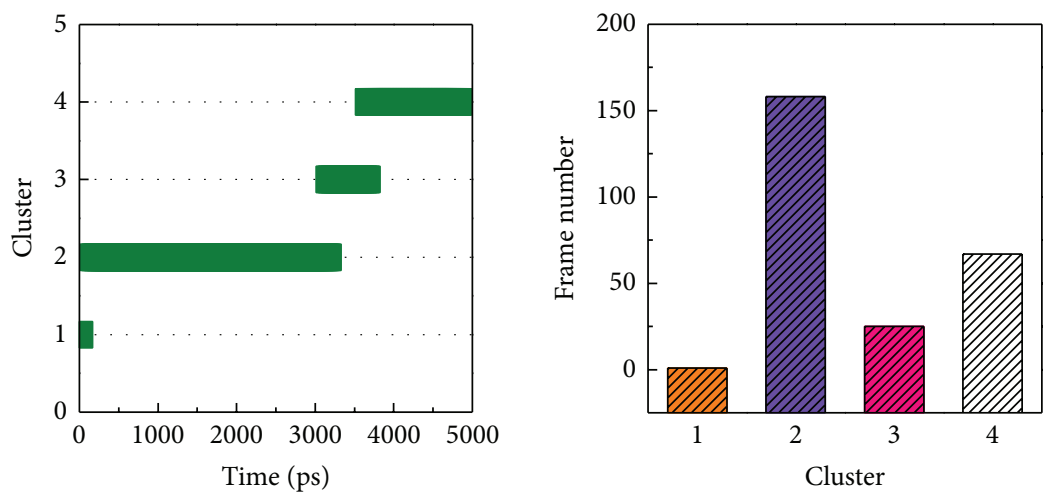

(d)

Figure 12: Clustering analyses of all conformations for a simulation time of 5,000 ps. 


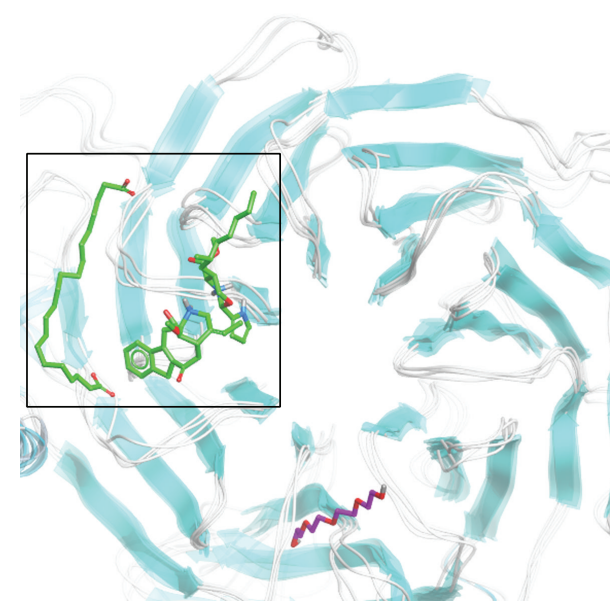

(a)
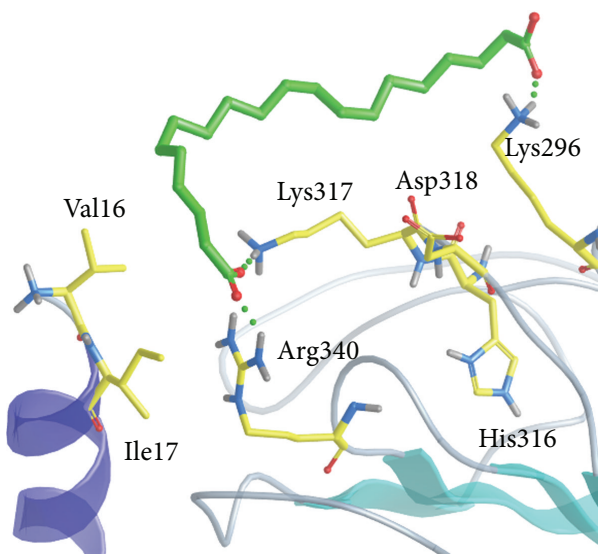

(c)

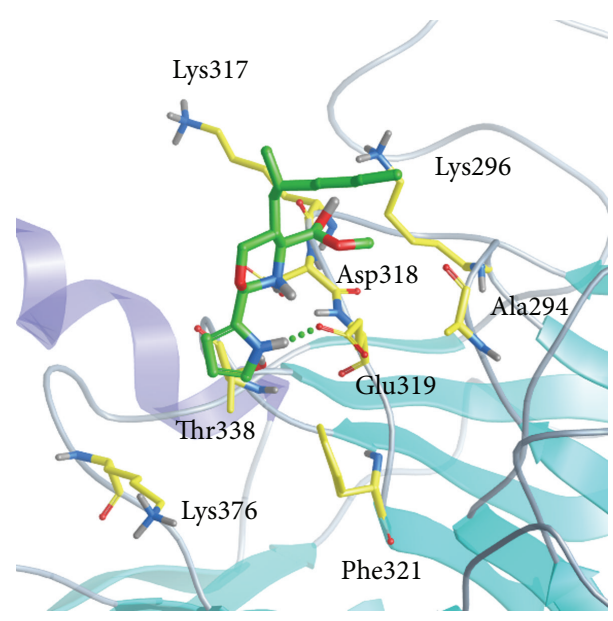

(b)

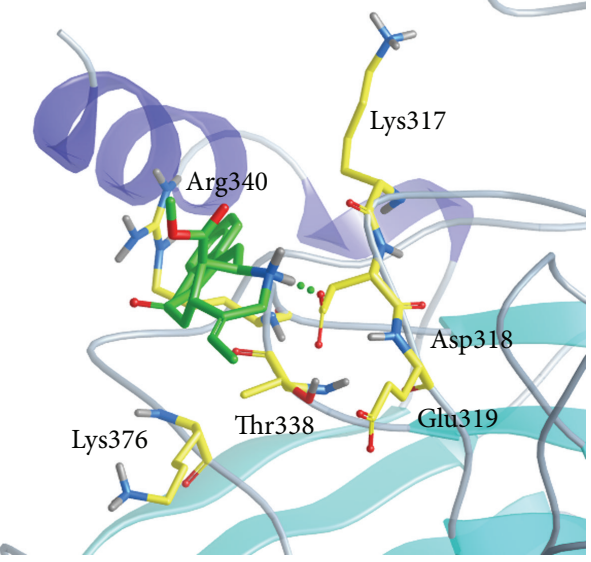

(d)

FIGURE 13: (a) Docking position of TCM candidates in superimposition of each representative conformation from MD simulation; the TCM compounds are colored in green. The docking poses of each TCM compound: (b) Bittersweet alkaloid II, (c) Eicosandioic acid, and (d) Perivine in RbAP48 binding site.

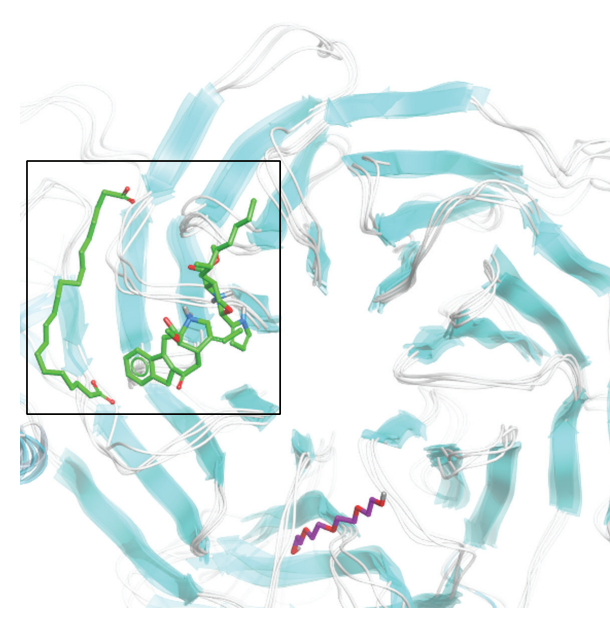

(a)

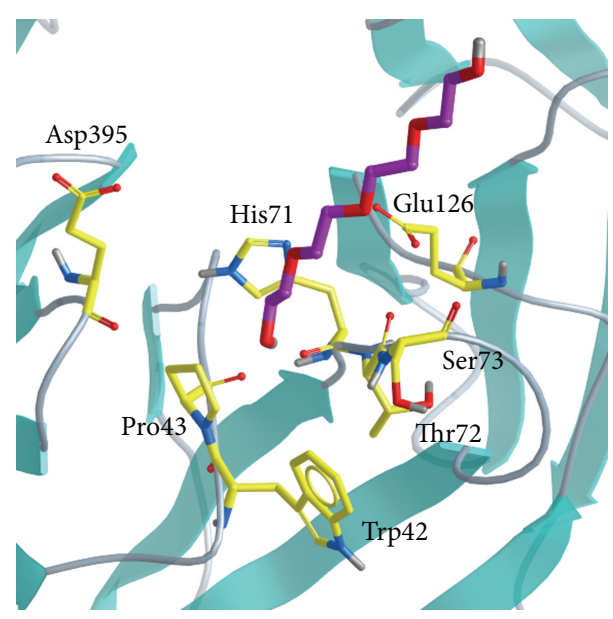

(b)

FIGURE 14: Docking position (a) and binding pose (b) of FOG1 in superimposition conformation. FOG1 is colored purple. 


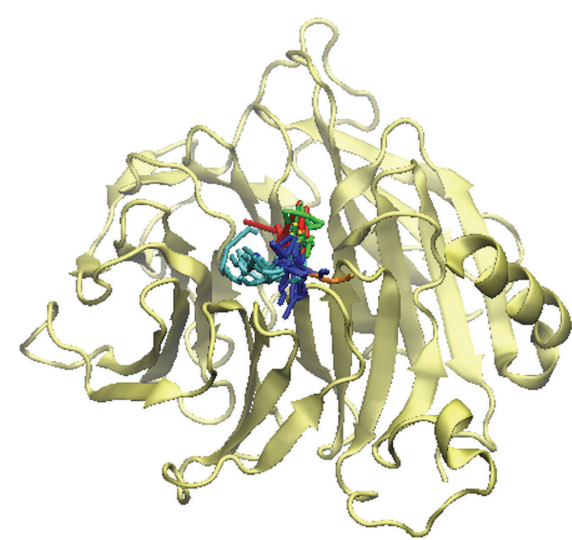

(a)

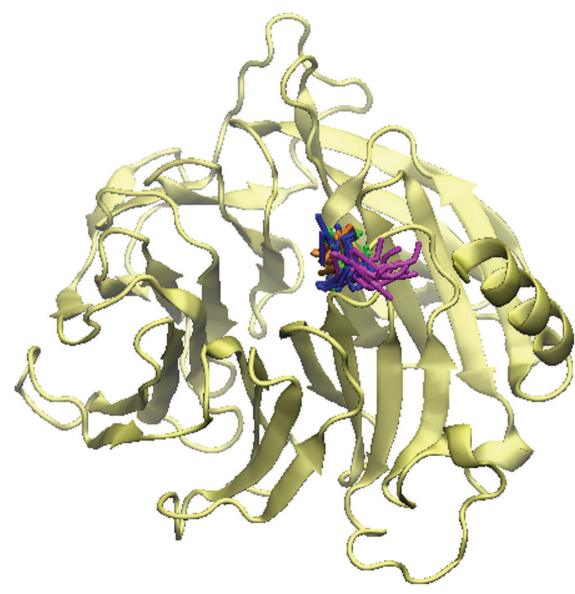

(c)

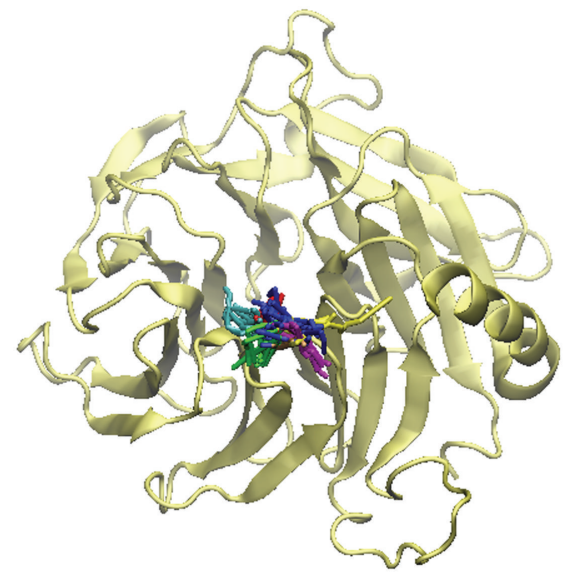

(b)

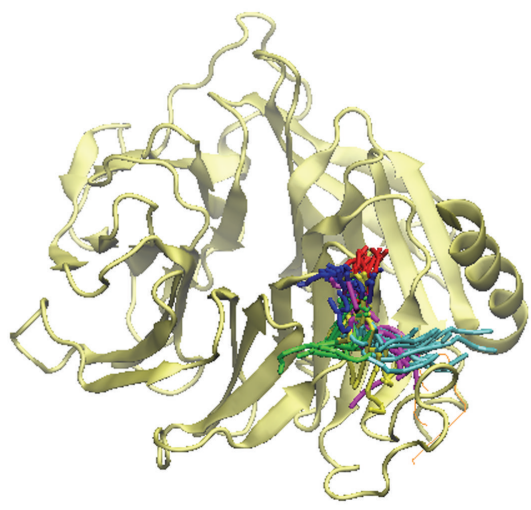

(d)

FIGURE 15: Ligand channel prediction of docked ligand in RbAp48: (a) Bittersweet alkaloid II (b) Eicosandioic acid, (c) Perivine, and (d) FOG1.

have a large protein-to-ligand distance of 500 to 3,000 ps (Figure 11), indicating that FOG1 was moving away from the initial binding position and transferring to another site in the protein structure. For the three TCM compounds, there were no substantial fluctuations in movement, suggesting that each ligand could bind stably in the RbAp48 structure.

3.5. Snapshots Analysis and Ligand Channel Prediction. In order to identify the most stable structure during the entire MD simulation and to understand the movement of FOG1, all conformations from MD simulation were clustered into three or four groups (Figure 12). The middle conformation from the final groups of clusters was chosen, and each middle frame is listed in Table 2 . The protein structures were then superimposed on each middle frame (Figure 13(a)). The position of FOG1 was found to be far from other three candidates due to the other three candidates not migrating significantly, but remaining close to the initial docking positions. The three candidates have common residues for ligand binding, Bittersweet alkaloid II generated one $\mathrm{H}$ bond with E319 (Figure 13(b)), Eicosandioic acid had two
H-bonds interacting with Arg340 and Lys276 (Figure 13(c)), and Perivine had one H-bond with Asp318 (Figure 13(d)). We found that K317, D318, and E319 can form H-bonds with the TCM candidates. In the initial docking poses, K317, D318, and E319 interacted with TCM compounds and FOG1, which illustrates that the docked ligands are not variable after MD simulation. From the FOG1 snapshot analysis, it can be seen that the docked ligand migrates significantly from the initial pose to the other site on RbAp48 (Figure 14(a)). Lys296 forms $\mathrm{H}$-bonds with FOG1 in the initial docking pose, but the surrounding residues changed to Glu395, His71, Pro43, Trp42, Glu126, Ser73, and Thr72 in the representative snapshot (Figure 14(b)). These results show that FOG1 is relatively more flexible than the other TCM compounds. In addition, we also predicted migration channel of each docked ligand during simulation time of 5000 ps; the prediction results were shown in Figure 15. The prediction of FOG1 displayed long distance channel than other three TCM compounds; the funding is correlated with snapshots analysis and migration analysis and illustrated that the TCM candidates could form stable binding conformation to interact with RbAp48. 
TABLE 2: Time of middle structure in each cluster from all MD conformations.

\begin{tabular}{|c|c|c|c|c|}
\hline \multirow{2}{*}{ Cluster } & \multicolumn{4}{|c|}{ Time of middle frame (ps) } \\
\hline & Bittersweet alkaloid II & Eicosandioic acid & Perivine & FOG1 \\
\hline 1 & 2320 & 1760 & 0 & 0 \\
\hline 2 & 3420 & 4380 & 320 & 2000 \\
\hline 3 & 3820 & 4980 & 2300 & 3400 \\
\hline 4 & 5000 & - & - & 4480 \\
\hline
\end{tabular}

\section{Conclusion}

From ADMET and docking analysis, our candidates are determined to be more drugs-like than FOG1, and the three scoring functions -PMF, -PMF04, and Dock Score are higher than the control. In migration analysis after $\mathrm{MD}$ simulation, FOG1 displayed low stability for RbAp48 binding, which was correlated with the low affinity in the docking results. The structure of RbAp48 did not change significantly during MD simulation, suggesting that FOG1 migration was not effected by protein structure. The unstable RbAp48-FOG1 complex could reduce the transcription function. The top three candidates Bittersweet alkaloid II, Eicosandioic acid, and Perivine bound stably in the binding site of RbAp48 and did not change the binding positions from the initial docking poses. Our results indicate that these TCM compounds may have potential for the design of novel drugs to solve the unstable RbAp48-FOG1 complex problem and provide a new mechanism for $\mathrm{AD}$ therapy.

\section{Conflict of Interests}

The authors declare that there is no conflict of interests regarding the publication of this paper.

\section{Acknowledgments}

The research was supported by Grants from the National Science Council of Taiwan (NSC102-2325-B039-001, NSC102-2221-E-468-027-), Asia University (ASIA100-CMU2, ASIA101-CMU-2, 102-ASIA-07), and China Medical University Hospital (DMR-102-051, DMR-103-058, DMR103-001, DMR-103-096). This study is also supported in part by Taiwan Department of Health Clinical Trial and Research Center of Excellence (DOH102-TD-B-111-004) and Taiwan Department of Health Cancer Research Center of Excellence (MOHW103-TD-B-111-03), and CMU under the Aim for Top University Plan of the Ministry of Education, Taiwan.

\section{References}

[1] R. L. Nussbaum and C. E. Ellis, "Alzheimer's disease and Parkinson's disease," The New England Journal of Medicine, vol. 348, no. 14, pp. 1356-1364, 2003.

[2] W. Thies and L. Bleiler, "2012 Alzheimer's disease facts and figures," Alzheimer's and Dementia, vol. 8, no. 2, pp. 131-168, 2012.

[3] B. Lam, M. Masellis, M. Freedman, D. T. Stuss, and S. E. Black, "Clinical, imaging, and pathological heterogeneity of the Alzheimer's disease syndrome," Alzheimer's Research and Therapy, vol. 5, no. 1, article 1, 2013.

[4] H. Oakley, S. L. Cole, S. Logan et al., "Intraneuronal $\beta$-amyloid aggregates, neurodegeneration, and neuron loss in transgenic mice with five familial Alzheimer's disease mutations: potential factors in amyloid plaque formation," Journal of Neuroscience, vol. 26, no. 40, pp. 10129-10140, 2006.

[5] P. Tiraboschi, L. A. Hansen, L. J. Thal, and J. Corey-Bloom, “The importance of neuritic plaques and tangles to the development and evolution of AD," Neurology, vol. 62, no. 11, pp. 1984-1989, 2004.

[6] R. Yaari and J. Corey-Bloom, "Alzheimer's disease," Seminars in Neurology, vol. 27, no. 1, pp. 32-41, 2007.

[7] W. Thies and L. Bleiler, "2013 Alzheimer's disease facts and figures," Alzheimer's \& Dementia, vol. 9, no. 2, pp. 208-245, 2013.

[8] M.-C. Yin, "Anti-glycative potential of triterpenes: a minireview," BioMedicine, vol. 2, no. 1, pp. 2-9, 2012.

[9] E. Pavlopoulos, S. Jones, S. Kosmidis et al., "Molecular mechanism for age-related memory loss: the histone-binding protein RbAp48," Science Translational Medicine, vol. 5, no. 200, Article ID 200ral15, 2013.

[10] S. A. Denslow and P. A. Wade, "The human Mi-2/NuRD complex and gene regulation," Oncogene, vol. 26, no. 37, pp. 5433-5438, 2007.

[11] N. J. Bowen, N. Fujita, M. Kajita, and P. A. Wade, "Mi2/NuRD: multiple complexes for many purposes," Biochimica et Biophysica Acta: Gene Structure and Expression, vol. 1677, no. 1-3, pp. 52-57, 2004.

[12] S.-C. Yang, S.-S. Chang, and C. Y.-C. Chen, "Identifying HER2 inhibitors from natural products database," PLoS ONE, vol. 6, no. 12, Article ID e28793, 2011.

[13] C.-L. Hsieh, "Acupuncture as treatment for nervous system diseases," BioMedicine, vol. 2, no. 2, pp. 51-57, 2012.

[14] K.-C. Chen, K.-W. Chang, H.-Y. Chen, and C. Y.-C. Chen, "Traditional Chinese medicine, a solution for reducing dual stroke risk factors at once?" Molecular BioSystems, vol. 7, no. 9, pp. 2711-2719, 2011.

[15] S.-C. Hsu and J.-G. Chung, "Anticancer potential of emodin," BioMedicine, vol. 2, no. 3, pp. 108-116, 2012.

[16] C. Y.-C. Chen, "A novel integrated framework and improved methodology of computer-aided drug design," Current Topics in Medicinal Chemistry, vol. 13, no. 9, pp. 965-988, 2013.

[17] H.-J. Huang, H. W. Yu, C.-Y. Chen et al., "Current developments of computer-aided drug design," Journal of the Taiwan Institute of Chemical Engineers, vol. 41, no. 6, pp. 623-635, 2010.

[18] C. Y.-C. Chen and W. I. Tou, "How to design a drug for the disordered proteins?” Drug Discovery Today, vol. 18, no. 19-20, pp. 910-915, 2013.

[19] K.-W. Chang, T.-Y. Tsai, K.-C. Chen et al., "iSMART: an integrated cloud computing web server for traditional Chinese 
medicine for online virtual screening, de novo evolution and drug design," Journal of Biomolecular Structure and Dynamics, vol. 29, no. 1, pp. 243-250, 2011.

[20] T.-Y. Tsai, K.-W. Chang, and C. Y.-C. Chen, "IScreen: world's first cloud-computing web server for virtual screening and de novo drug design based on TCM Database@Taiwan," Journal of Computer-Aided Molecular Design, vol. 25, no. 6, pp. 525-531, 2011.

[21] C.-C. Li, H.-Y. Lo, C.-Y. Hsiang, and T.-Y. Ho, "DNA microarray analysis as a tool to investigate the therapeutic mechanisms and drug development of Chinese medicinal herbs," BioMedicine, vol. 2, no. 1, pp. 10-16, 2012.

[22] H. J. Huang, Y. R. Jian, and C. Y.-C. Chen, "Traditional Chinese medicine application in HIV: an in silico study," Journal of Biomolecular Structure and Dynamics, vol. 32, no. 1, pp. 1-12, 2014.

[23] G. Y. Yang, H. Luo, X. Liao, and J. P. Liu, "Chinese herbal medicine for the treatment of recurrent miscarriage: a systematic review of randomized clinical trials," BMC Complementary and Alternative Medicine, vol. 13, article 320, 2013.

[24] P.-C. Lin, P.-Y. Liu, S.-Z. Lin, and H.-J. Harn, "Angelica sinensis: a Chinese herb for brain cancer therapy," BioMedicine, vol. 2, no. 1, pp. 30-35, 2012.

[25] S.-C. Hsu, J.-H. Lin, S.-W. Weng et al., "Crude extract of Rheum palmatum inhibits migration and invasion of U-2 OS human osteosarcoma cells by suppression of matrix metalloproteinase2 and -9," BioMedicine, vol. 3, no. 3, pp. 120-129, 2013.

[26] S.-S. Chang, H.-J. Huang, and C. Y.-C. Chen, "Two birds with one stone? Possible dual-targeting H1N1 inhibitors from traditional Chinese medicine," PLoS Computational Biology, vol. 7, no. 12, Article ID e1002315, 2011.

[27] S.-S. Chang, H.-J. Huang, and C. Y.-C. Chen, "High performance screening, structural and molecular dynamics analysis to identify H1 inhibitors from TCM Database@Taiwan," Molecular BioSystems, vol. 7, no. 12, pp. 3366-3374, 2011.

[28] T.-T. Chang, M.-F. Sun, H.-Y. Chen et al., "Screening from the world's largest TCM database against H1N1 virus," Journal of Biomolecular Structure and Dynamics, vol. 28, no. 5, pp. 773786, 2011.

[29] T.-T. Chang, K.-C. Chen, K.-W. Chang et al., "In silico pharmacology suggests ginger extracts may reduce stroke risks," Molecular BioSystems, vol. 7, no. 9, pp. 2702-2710, 2011.

[30] K.-C. Chen and C. Y.-C. Chen, "Stroke prevention by traditional Chinese medicine? A genetic algorithm, support vector machine and molecular dynamics approach," Soft Matter, vol. 7, no. 8, pp. 4001-4008, 2011.

[31] C. Y.-C. Chen, "Computational screening and design of Traditional Chinese Medicine (TCM) to block phosphodiesterase-5," Journal of Molecular Graphics and Modelling, vol. 28, no. 3, pp. 261-269, 2009.

[32] K.-C. Chen, S.-S. Chang, H.-J. Huang, T.-L. Lin, Y.-J. Wu, and C. Y.-C. Chen, "Three-in-one agonists for PPAR-a, PPAR- $\gamma$, and PPAR-d from traditional Chinese medicine," Journal of Biomolecular Structure and Dynamics, vol. 30, no. 6, pp. 662683, 2012.

[33] K.-Y. Chen, S.-S. Chang, and C. Y.-C. Chen, "In silico identification of potent pancreatic triacylglycerol lipase inhibitors from traditional Chinese medicine," PLoS ONE, vol. 7, no. 9, Article ID e43932, 2012.

[34] K.-C. Chen, S. S. Chang, F. J. Tsai, and C. Y.-C. Chen, "Han ethnicity-specific type 2 diabetic treatment from traditional
Chinese medicine?" Journal of Biomolecular Structure and Dynamics, vol. 31, no. 11, pp. 1219-1235, 2013.

[35] K.-C. Chen, Y. R. Jian, M. F. Sun et al., "Investigation of silent information regulator 1 (Sirt1) agonists from Traditional Chinese Medicine," Journal of Biomolecular Structure and Dynamics, vol. 31, no. 11, pp. 1207-1218, 2013.

[36] K.-C. Chen, M.-F. Sun, S.-C. Yang et al., "Investigation into potent inflammation inhibitors from traditional Chinese medicine," Chemical Biology and Drug Design, vol. 78, no. 4, pp. 679-688, 2011.

[37] H.-J. Huang, C.-C. Lee, and C. Y.-C. Chen, "Pharmacological chaperone design for reducing risk factor of Parkinson's disease from traditional Chinese medicine," Evidence-Based Complementary and Alternative Medicine, vol. 2014, Article ID 830490, 12 pages, 2014.

[38] Y.-A. Tsou, K.-C. Chen, H.-C. Lin, S.-S. Chang, and C. Y.-C. Chen, "Uroporphyrinogen decarboxylase as a potential target for specific components of traditional Chinese medicine: a virtual screening and molecular dynamics study," PLOS ONE, vol. 7, no. 11, Article ID e50087, 2012.

[39] S.-C. Yang, S.-S. Chang, H.-Y. Chen, and C. Y.-C. Chen, "Identification of potent EGFR inhibitors from TCM Database@Taiwan," PLoS Computational Biology, vol. 7, no. 10, Article ID e1002189, 2011.

[40] C. Y.-C. Chen, “TCM Database@Taiwan: the world's largest traditional Chinese medicine database for drug screening in silico," PLoS ONE, vol. 6, no. 1, Article ID e15939, 2011.

[41] S. Lejon, S. Y. Thong, A. Murthy et al., "Insights into association of the NuRD complex with FOG-1 from the crystal structure of an RbAp48.FOG-1 complex," Journal of Biological Chemistry, vol. 286, no. 2, pp. 1196-1203, 2011.

[42] Accelerys, Discovery Studio Client V2.5, Accelrys, San Diego, Calif, USA, 2009.

[43] B. Xue, R. L. Dunbrack, R. W. Williams, A. K. Dunker, and V. N. Uversky, "PONDR-FIT: a meta-predictor of intrinsically disordered amino acids," Biochimica et Biophysica Acta: Proteins and Proteomics, vol. 1804, no. 4, pp. 996-1010, 2010.

[44] S. Pronk, S. Páll, R. Schulz et al., "GROMACS 4.5: a highthroughput and highly parallel open source molecular simulation toolkit," Bioinformatics, vol. 29, no. 7, pp. 845-854, 2013.

[45] V. Zoete, M. A. Cuendet, A. Grosdidier, and O. Michielin, "SwissParam: a fast force field generation tool for small organic molecules," Journal of Computational Chemistry, vol. 32, no. 11, pp. 2359-2368, 2011.

[46] W. I. Tou and C. Y.-C. Chen, "May disordered protein cause serious drug side effect?" Drug Discovery Today, vol. 19, no. 4, pp. 367-372, 2014. 

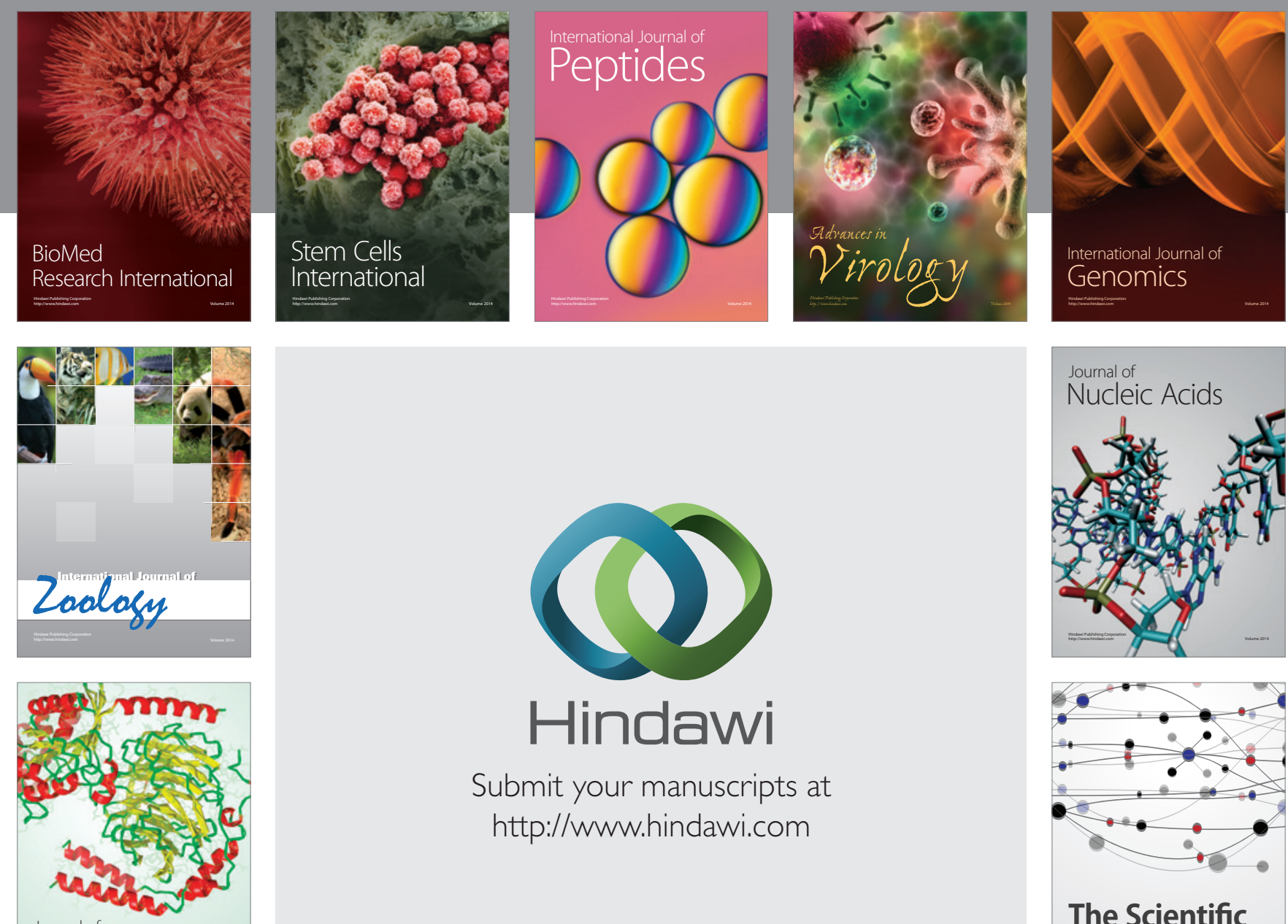

Submit your manuscripts at

http://www.hindawi.com

Journal of
Signal Transduction
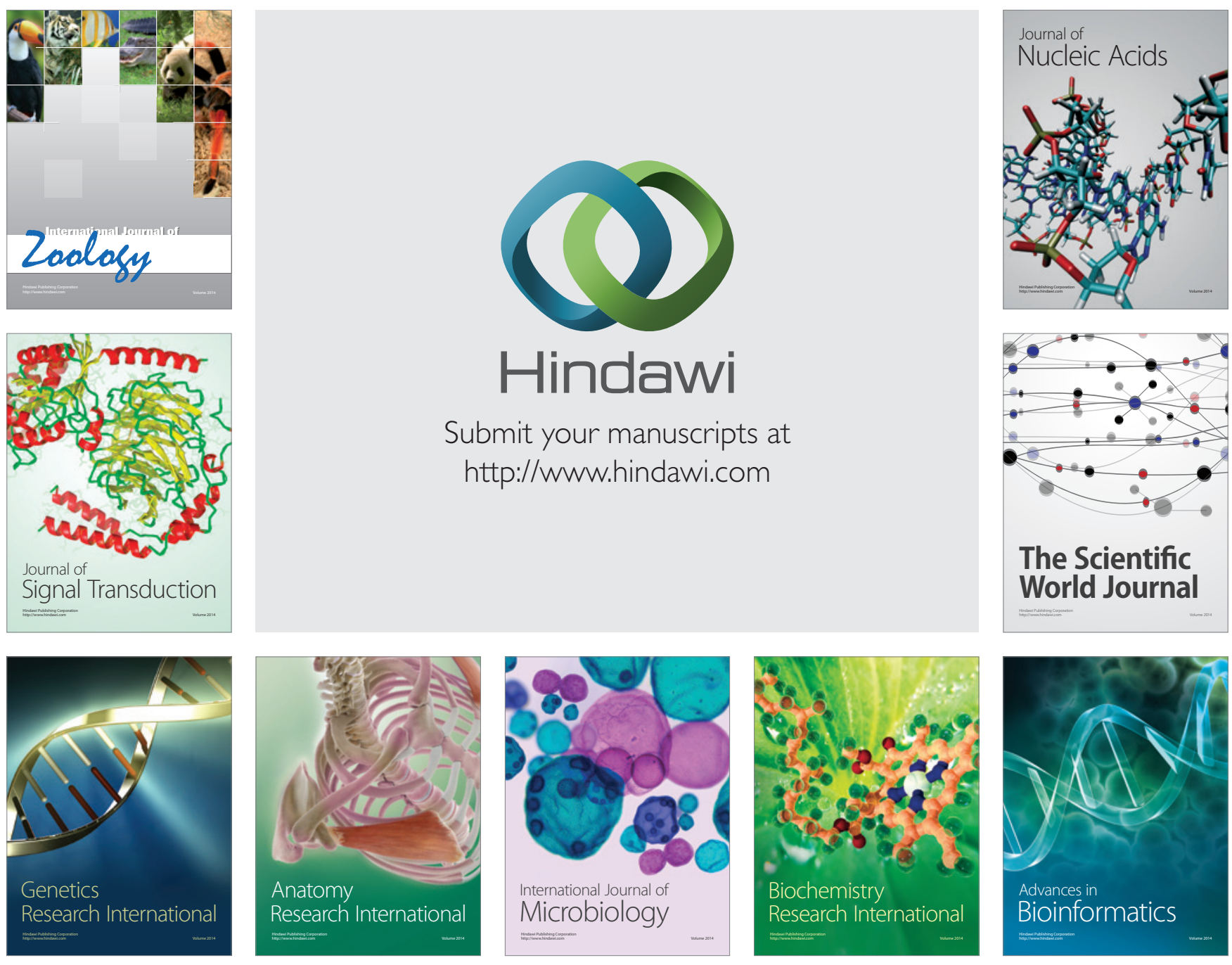

The Scientific World Journal
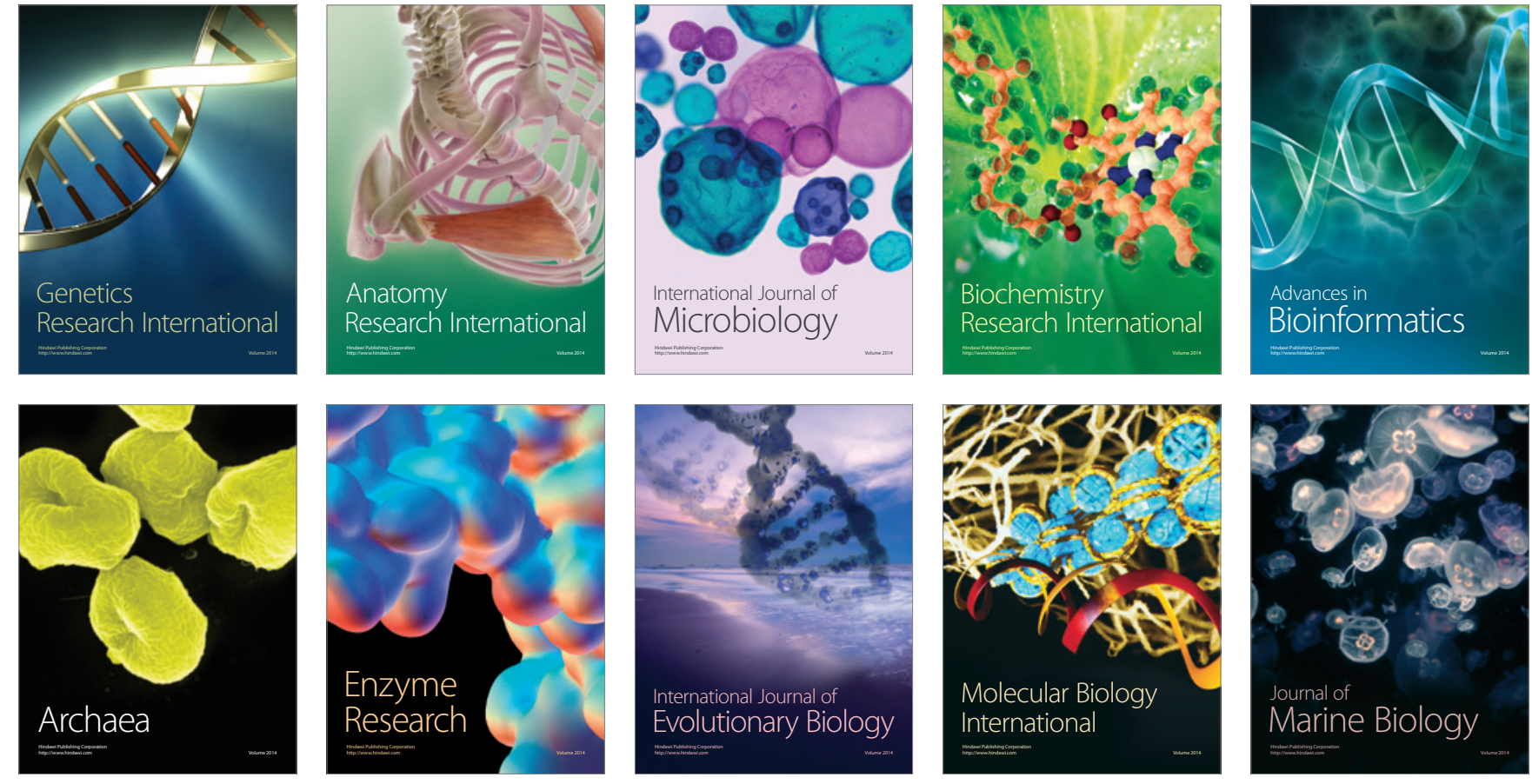